\title{
Direct Self-Assembly of Conductive Nanorods of Metal- Organic Frameworks into Chemiresistive Devices on Shrinkable Polymer Films
}

\author{
Merry K. Smith, Kennedy E. Jensen, Polina A. Pivak, and Katherine A. Mirica* \\ Dartmouth College, Department of Chemistry \\ Burke Laboratory, Hanover, NH 03755, USA
}

Email: katherine.a.mirica@dartmouth.edu, phone: 603-646-8188

\section{SUPPORTING INFORMATION}

I. General Methods

(21 pages)

II. Templated and General Synthesis of Compounds

BULK MOFs

III. Powder X-Ray Diffraction of MOFs

S4

IV. Scanning Electron Microscopy of MOFs

S5

V. Energy Dispersive X-Ray Spectroscopy of MOFs S6

VI. Thermal Gravimetric Analysis of MOFs

ELECTRODES

VII. Quantitative Analysis of Shrinkable Graphitic Electrodes $\quad$ S7

VIII. Interferometry of Electrodes

IX. 4-Point Linear Probe Measurement of Resistivity

FULL DEVICES

X. Current/Voltage Plots

XI. Scanning Electron Microscopy of Devices

XII. Energy Dispersive X-Ray Spectroscopy of Devices

S15

XIV. Interferometry of Devices

S16

XV. Variance Device:Device and Batch:Batch $\quad$ S17

XVI. Chemiresistive Sensing Results

XVII. Principle Component Analysis

XVIII. Infrared Analysis of Shrinkable Polymer Films

XIX. Supporting References 


\section{General Methods}

Chemicals were purchased from Sigma Aldrich or $\mathrm{TCl}$ and used as received. Powder X-ray diffraction (PXRD) data was gathered using a Bruker D8 diffractometer. Spectra are presented with the background subtracted and corrected for K- $\alpha$. Scanning Electron Microscopy (SEM) and Energy Dispersive X-ray Spectroscopy (EDS) was performed using a Hitachi TM3000 SEM equipped for X-ray microanalysis with a Bruker Edax light element $\mathrm{Si}(\mathrm{Li})$ detector. We obtained Thermal Gravimetric Analysis (TGA) traces using a TA instruments TGA Q50 with platinum pans. Infrared spectra were recorded using a Jacso FT/IR-6200 equipped with a Jasco ATR PRO450-S. Commercial shrinkable Grafix@ brand polystyrene-based polymer films were purchased from Amazon and used as received. ${ }^{\mathrm{S} 1}$ Custom tracers were prepared for the tracing of drawn devices, shown in Figure $\mathbf{S 1 .}$

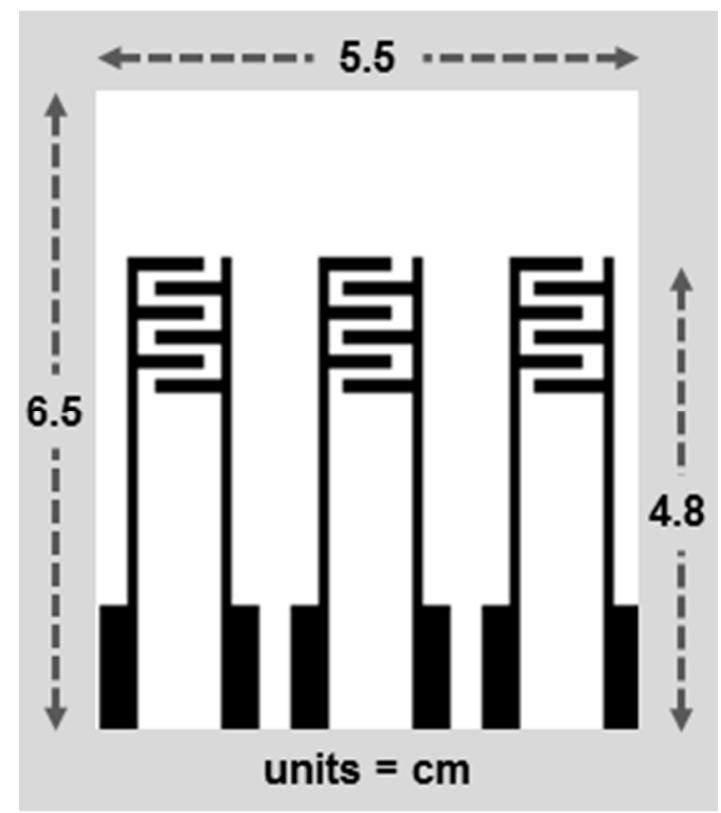

Figure S1. Optimized custom electrode tracer, to scale.

We prepared a $0.033 \mathrm{M}$ solution of 2,3,5,6,10,11-hexahydroxytriphenylene (HHTP) mixed with metal salt (2 molar equivalents) in deionized water in a beaker, and suspended the polymeric device chips equipped with interdigitated graphitic electrodes in the solution (Figure 1D). We masked areas that would be submerged in MOF solution, but where MOF growth was not desired, using clear nail polish. The simplicity of this methodology enables rapid prototyping of different architectures of electrodes for devices or rapid fabrication of multiple indistinguishable devices on the same chip (Figure 2A).

Conductive thin films of $\mathrm{M}_{3} \mathrm{HHTP}_{2}(\mathrm{M}=\mathrm{Cu}$ or Ni) MOFs self-assembled on the surface of the devices (see section II) overnight upon stirring in a sealed beaker under air at $85^{\circ} \mathrm{C}$. We rinsed the devices thoroughly with deionized (DI) water, then submerged them in DI water to for one hour to activate the MOFs and remove any residual starting materials and bi-products, then dried the activated devices. If the MOFs had grown on the masked areas, potentially bridging 
the electrodes of the adjacent devices, we used a spatula to remove MOF film from the masked areas. This processing step was more rapid than using a removable mask, such as tape.

\section{Templated and General Synthesis of Compounds}

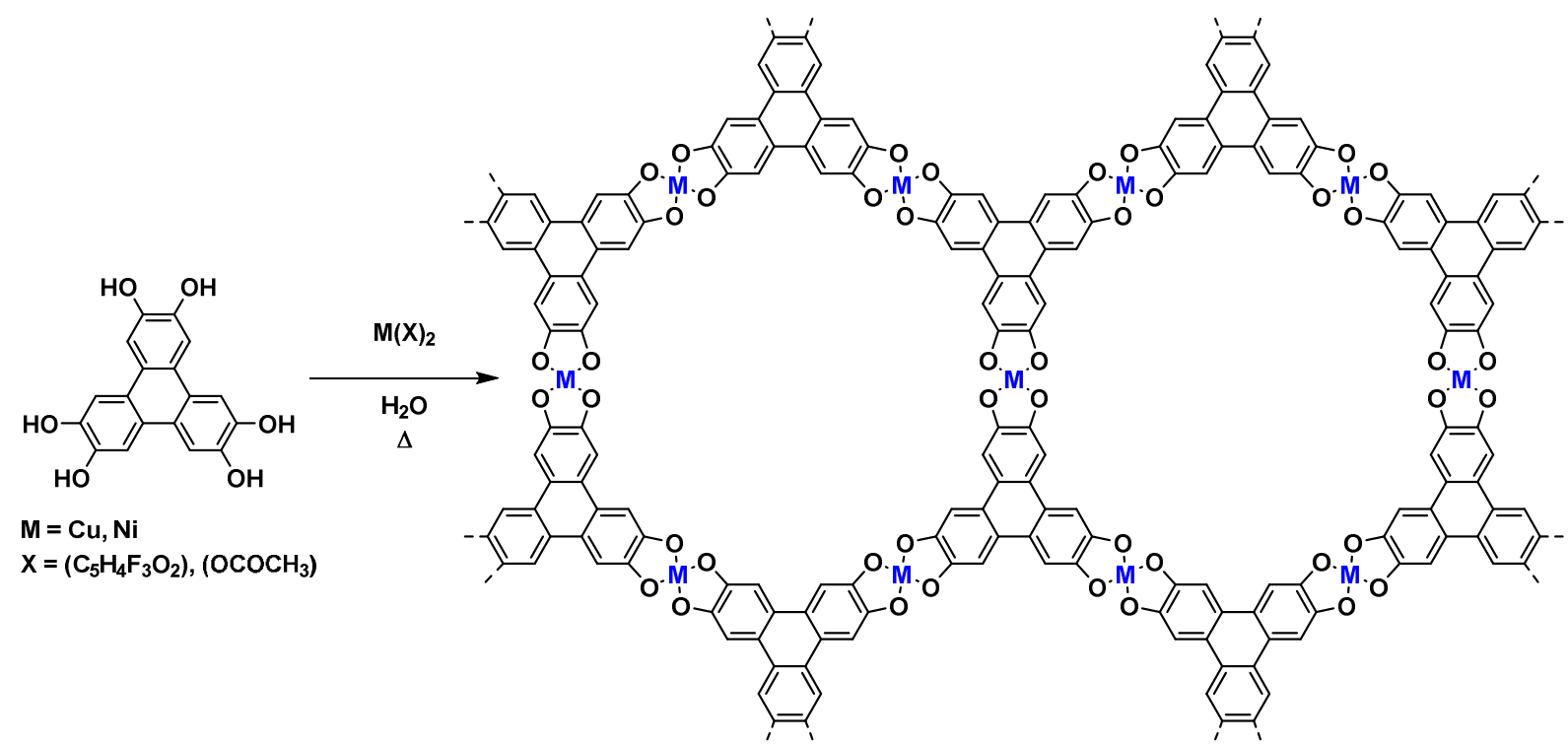

Scheme S1. General reaction scheme for the synthesis of metal organic frameworks $\mathrm{Cu}_{3} \mathrm{HHTP}_{2} \mathrm{MOF}$ and $\mathrm{Ni}_{3} \mathrm{HHTP}_{2}$ MOF.

Templated Synthesis of $\mathrm{Cu}_{3} \mathrm{HHTP}_{2}$ MOF on shrinkable polymeric films: Three chips were prepared from shrinkable films with graphite electrodes. Clear nail polish was used to mask areas where MOF growth was not desired. To prepare MOFs, 1.32 mmol 2,3,5,6,10,11hexahydroxytriphenylene and $2.64 \mathrm{mmol}$ copper (II) trifluoroacetylacetonate were added to a 25 $\mathrm{mL}$ beaker. Deionized water $(4 \mathrm{~mL})$ was added and the three chips with triplicate interdigitated electrodes were introduced to the reaction mixture such that the interdigitated portion was submerged at the solution meniscus. A strip of masking tape was used to both cover the reverse sides of the chips and to mount the chips within the beaker, such that the tape was looped over the edge of the beaker and secured on the outside of the flask. The beaker was capped with a watch glass and sealed with parafilm, and the reaction mixture subjected to sonication $(5 \mathrm{~min})$, then heated with stirring (solution at $80^{\circ} \mathrm{C}$ ) overnight. The resulting devices were allowed to cool, removed from the beaker, washed thoroughly with water $(2 \times 5 \mathrm{~mL})$, then submerged them DI water for one hour to activate the MOFs and remove any residual starting materials and bi-products. The resulting activated devices were dried in an oven at $160^{\circ} \mathrm{C}$ for 5 minutes. If MOFs had grown on the masked areas, bridging the electrodes of the adjacent devices, a spatula was used to remove MOF film from the masked areas.

Templated Synthesis of $\mathrm{Ni}_{3} \mathrm{HHTP}_{2}$ MOF on shrinkable polymeric film: Three chips were prepared from shrinkable films with graphite electrodes. Clear nail polish was used to mask areas where MOF growth was not desired. To prepare MOFs, 1.32 mmol 2,3,5,6,10,11hexahydroxytriphenylene and $2.64 \mathrm{mmol}$ nickel (II) acetate were added to a $25 \mathrm{~mL}$ beaker. Deionized water $(4 \mathrm{~mL})$ was added and the three chips with triplicate interdigitated electrodes were introduced to the reaction mixture such that the interdigitated portion was submerged at 
the solution meniscus. A strip of masking tape was used to both cover the reverse sides of the chips and to mount the chips within the beaker, such that the tape was looped over the edge of the beaker and secured on the outside of the flask. The beaker was capped with a watch glass and sealed with parafilm, and the reaction mixture subjected to sonication ( $5 \mathrm{~min}$ ), then heated with stirring (solution at $80^{\circ} \mathrm{C}$ ) overnight. The resulting devices were allowed to cool, removed from the beaker, washed thoroughly with water $(2 \times 5 \mathrm{~mL})$, then submerged them DI water for one hour to activate the MOFs and remove any residual starting materials and bi-products. The resulting activated devices were dried in an oven at $160^{\circ} \mathrm{C}$ for 5 minutes. If MOFs had grown on the masked areas, bridging the electrodes of the adjacent devices, a spatula was used to remove MOF film from the masked areas.

Analogous conditions were used to synthesize MOFs in solution for characterization. Reaction mixtures were filtered, solids rinsed with water and dried thoroughly under vacuum. Characterization matched reported analyses for PXRD (Figure S2), EDS (Figure S5), TGA (Figure S6), and bulk conductivity.

\section{Powder X-Ray Diffraction of MOFs}

Powder X-Ray Diffraction (PXRD) was performed using a Bruker D8 Diffractometer. Homogenized, flat power samples were analyzed in a $25 \mathrm{~mm}$ diameter zero diffraction PMMA sample holders ( $8.5 \mathrm{~mm}$ height, Bruker, $\sim 0.5 \mathrm{~g}$ sample size).

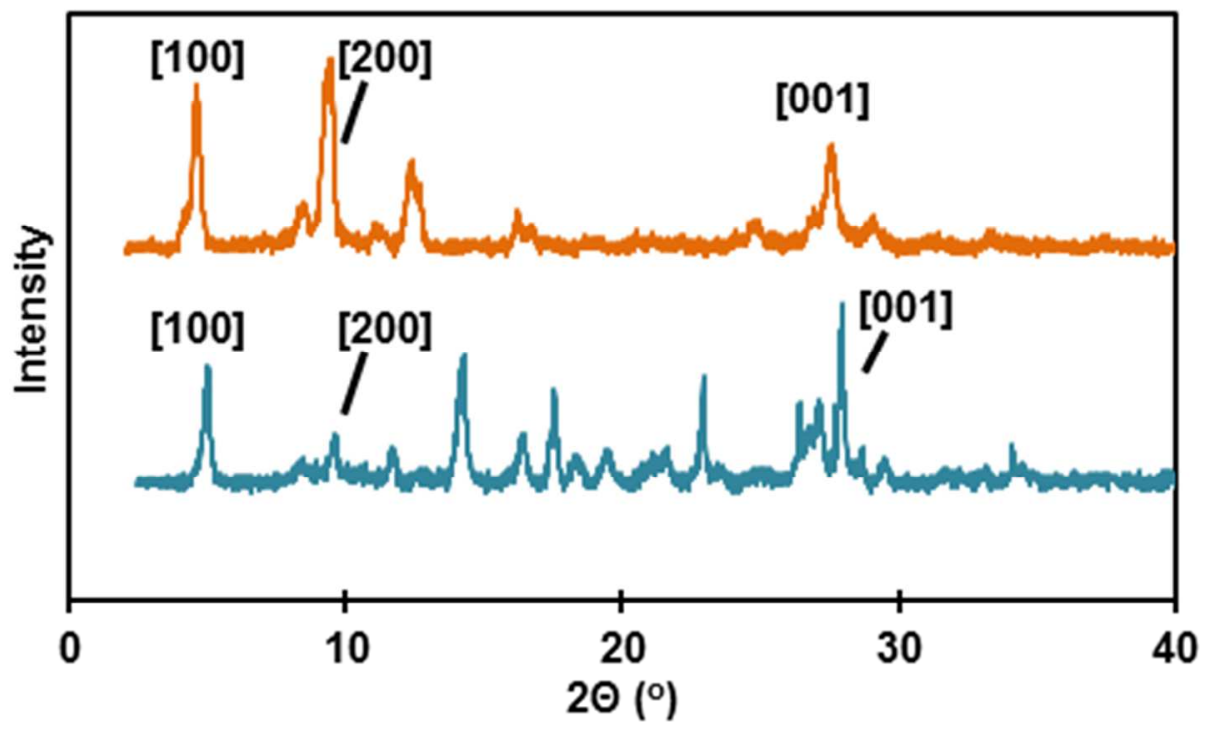

Figure S2. PXRD traces for bulk $\mathrm{Cu}_{3} \mathrm{HHTP}_{2} \mathrm{MOF}$ (orange) and $\mathrm{Ni}_{3} \mathrm{HHTP}_{2}$ MOF (blue). 


\section{Scanning Electron Microscopy of MOFs}

Scanning electron microscopy of bulk MOFs was obtained using a using a Hitachi TM3000 SEM with a $15.0 \mathrm{kV}$ beam.
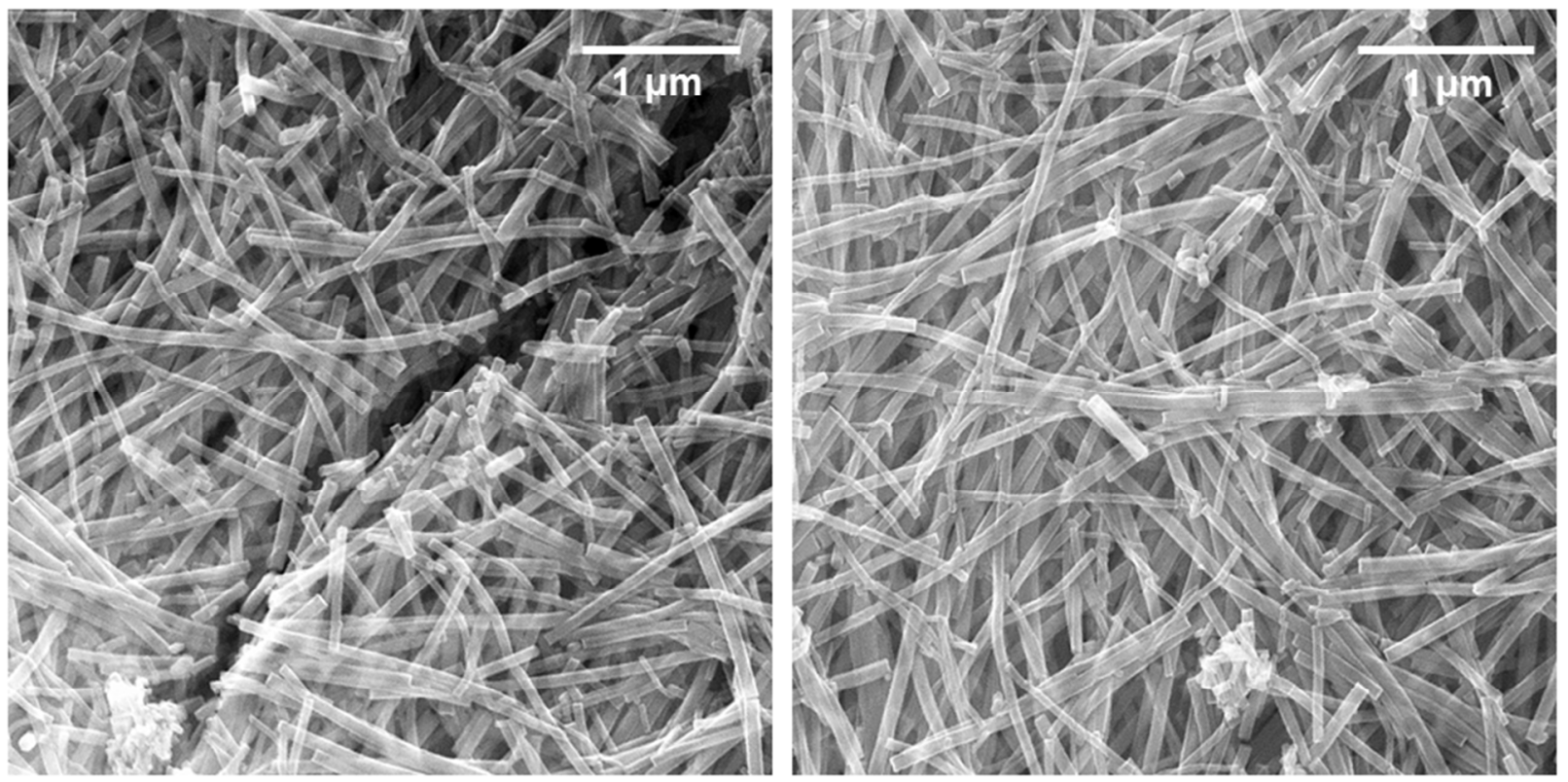

Figure S3. Scanning electron micrographs of bulk $\mathrm{Cu}_{3} \mathrm{HHTP}_{2} \mathrm{MOF}$ (20000x magnification) at multiple locations within the sample, illustrating the homogeneity of the crystallite diameters.
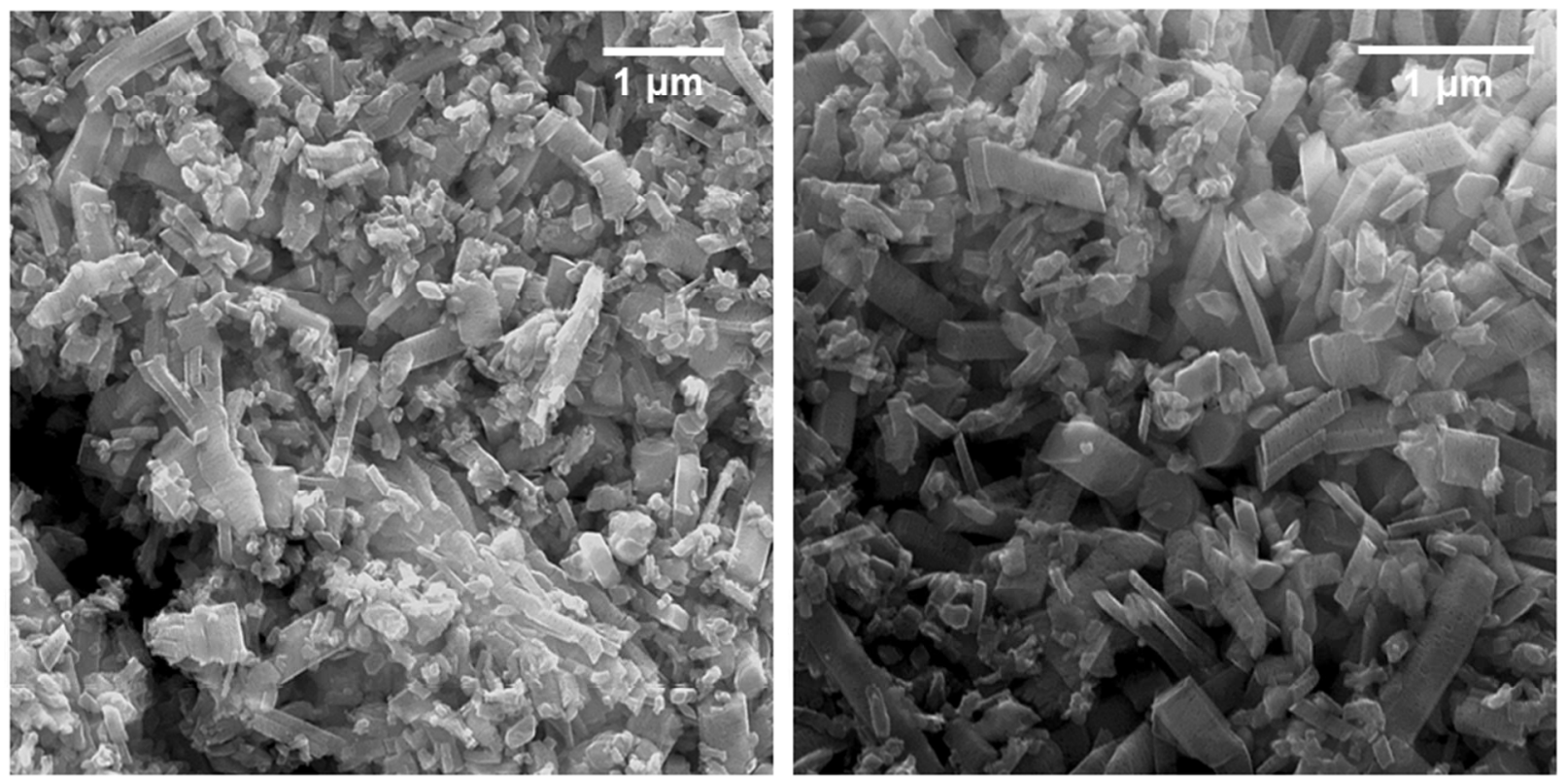

Figure S4. Scanning electron microscopy of bulk $\mathrm{Ni}_{3} \mathrm{HHTP}_{2}$ MOF at 16000x magnification (left) and 20000x magnification (right) at multiple locations within the sample, illustrating consistency in crystallite formation and morphology. 


\section{Energy Dispersive X-Ray Spectroscopy of MOFs}

Energy dispersive X-Ray spectroscopy was collected using SDD X-ray microanalysis system with Octane Pro 10 sq. $\mathrm{mm}$ detector and TEAM software.

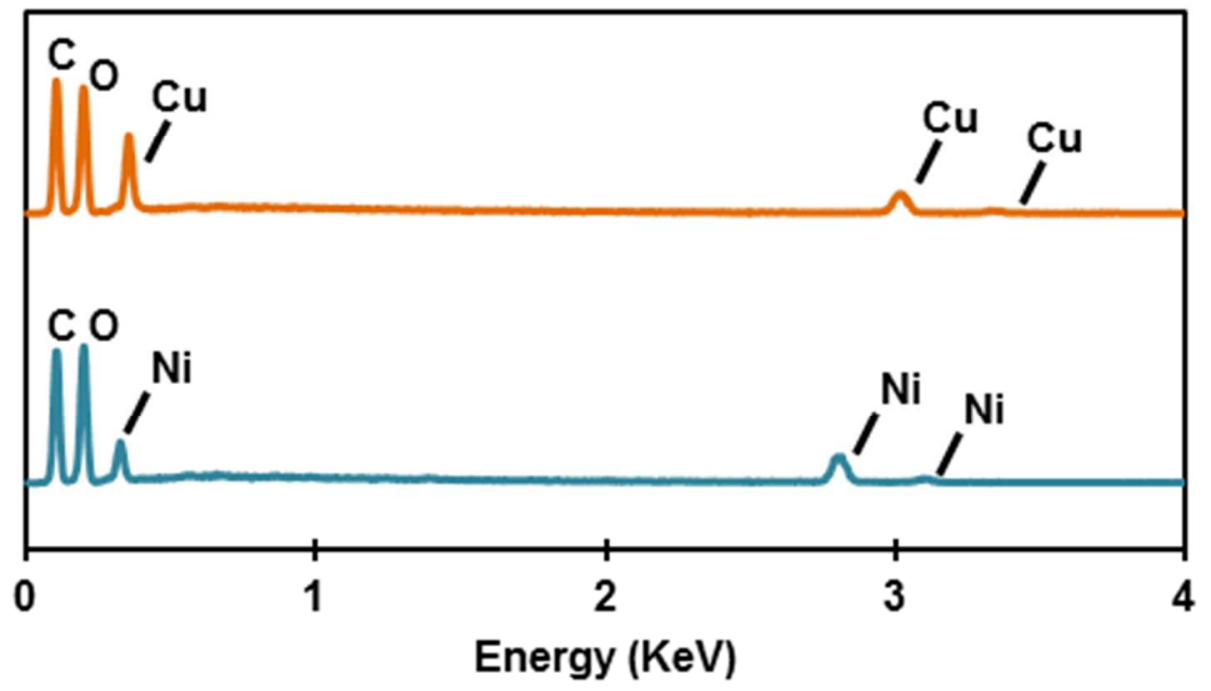

Figure S5. Energy dispersive spectroscopy of bulk $\mathrm{Cu}_{3} \mathrm{HHTP}_{2} \mathrm{MOF}$ (orange) and $\mathrm{Ni}_{3} \mathrm{HHTP}_{2} \mathrm{MOF}$ (blue).

\section{Thermal Gravimetric Analysis of MOFs}

Thermal gravimetric analysis was performed using a TA Instruments TGA Q150 with a 30 ${ }^{\circ} \mathrm{C} / \mathrm{min}$ ramp from room temperature to $900{ }^{\circ} \mathrm{C}$.
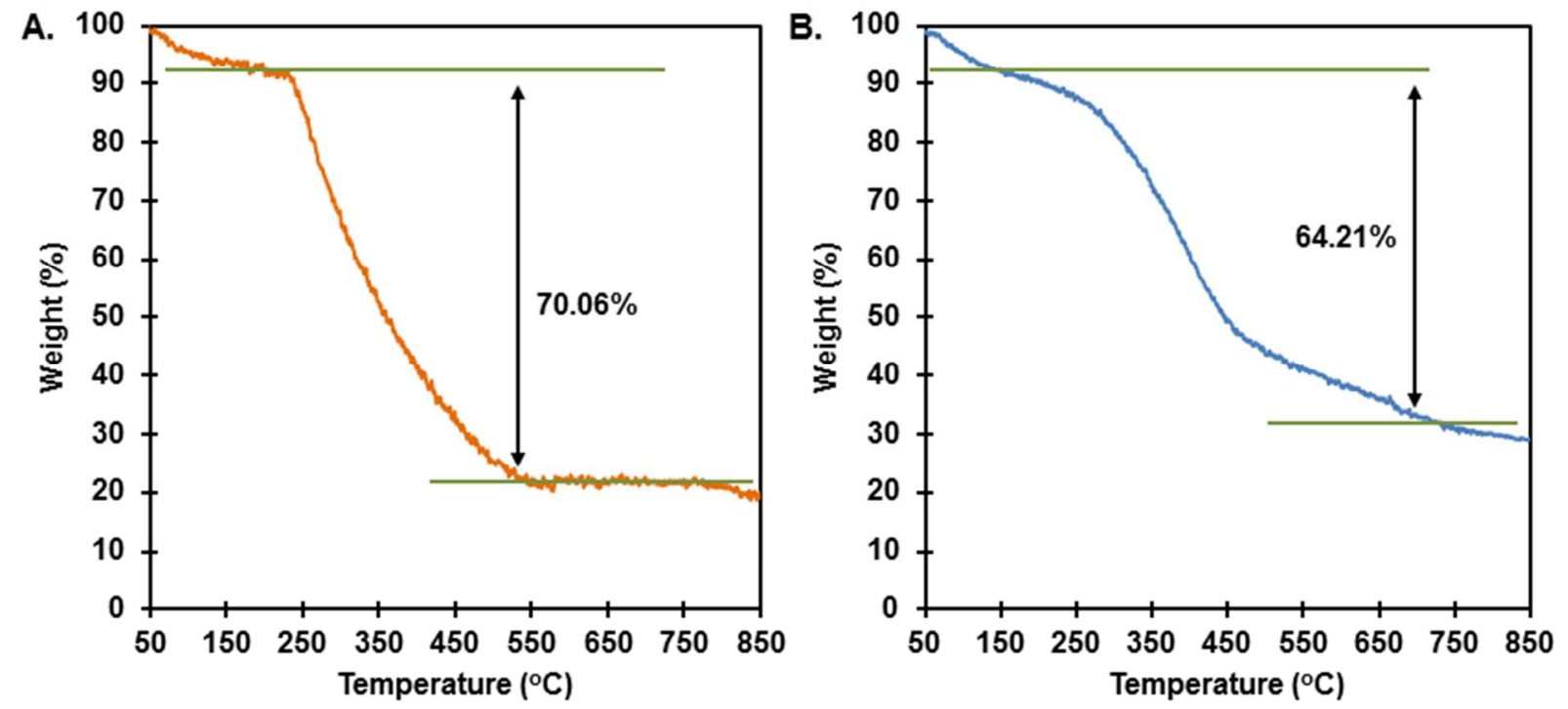

Figure S6. A) TGA curve of $\mathrm{Cu}_{3} \mathrm{HHTP}_{2}$ MOF filtered and dried from solution of templated device synthesis, B) TGA curve of $\mathrm{Ni}_{3} \mathrm{HHTP}_{2}$ MOF filtered and dried from solution of templated device synthesis. 


\section{Quantitative Analysis of Shrinkable Graphitic Electrodes}

\section{Inset from Figure 1B - All Data}

Polystyrene-based Grafix@ polymeric films ${ }^{\mathrm{S1}}$ (see Section XVIII) were used as the substrates for shrinkable electrodes. The $\mathrm{x}, \mathrm{y}$-dimensions (length and width) of wires before and after shrinking for both a sample device (Figure S7A) and actual electrode (Figure S7B) were measured using calipers. The z-dimension (thickness) for both devices was collected using a Zygo NewView 7300 Light Interferometer equipped with a 10x lens (Figures S8-S15). We obtained resistivity measurements using a two-point measurement with a multimeter. All values are reported in Table S1.

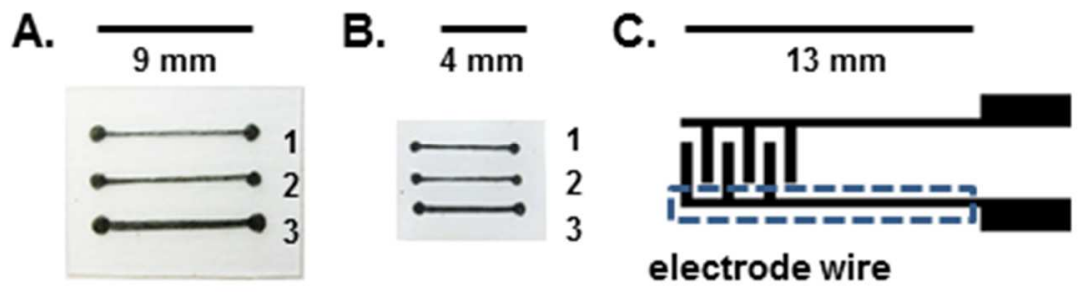

Figure S7. A) Photograph of graphitic wires with different widths drawn on top of a shrinkable polymeric film (view before shrinking. B) Photograph of same graphitic wires with different widths as shown in ' $A$ ' after shrinking the polymeric film substrate. C) Electrode tracer device (not to scale) demonstrating region of wire considered in this experiment.

Table S1. Wire Dimensions and Resistivity Before and After Shrinking. L= length of wire, $\mathrm{W}=$ width of wire, $\mathrm{T}=$ thickness of wire (interferometry).

\begin{tabular}{|c|c|c|c|c|}
\hline \multicolumn{5}{|c|}{ Before Shrinking } \\
\hline Wire & $\mathrm{L}(\mathrm{mm})$ & $\underset{(\mathrm{mm})}{W}$ & $\mathrm{~T}(\mu \mathrm{m})$ & $\begin{array}{l}\text { 2-point } \\
\text { Resistivity }\end{array}$ \\
\hline 1 & 9.02 & 0.30 & $<1^{a}$ & $1.016 \mathrm{M} \Omega$ \\
\hline 2 & 9.02 & 0.45 & $<1^{a}$ & $206.9 \mathrm{k} \Omega$ \\
\hline 3 & 8.96 & 0.64 & $<1^{a}$ & $97.9 \mathrm{k} \Omega$ \\
\hline Electrode & 35.4 & 1.08 & $<1^{a}$ & $59.9 \mathrm{k} \Omega$ \\
\hline \multicolumn{5}{|c|}{ After Shrinking } \\
\hline 1 & 3.79 & 0.08 & $6-10^{b}$ & $287.7 \mathrm{k} \Omega$ \\
\hline 2 & 3.79 & 0.16 & $\sim 10^{b}$ & $320.6 \mathrm{k} \Omega$ \\
\hline 3 & 3.64 & 0.39 & $\sim 10^{b}$ & $119.2 \mathrm{k} \Omega$ \\
\hline Electrode & 13.7 & 0.46 & $\sim 10^{b}$ & $33.76 \mathrm{k} \Omega$ \\
\hline
\end{tabular}

${ }^{a}$ True thickness is below $1 \mu \mathrm{m}$. When we abrade with a graphite pencil, the graphite fills in the lowest points of the polymer film surface profile, resulting in no measurable step-change. According to interferometer results, the graphitic wire thickness is less than $1 \mu \mathrm{m}$ before shrinking. ${ }^{\mathrm{b}}$ Approximate values. 


\section{Interferometry of Electrodes}

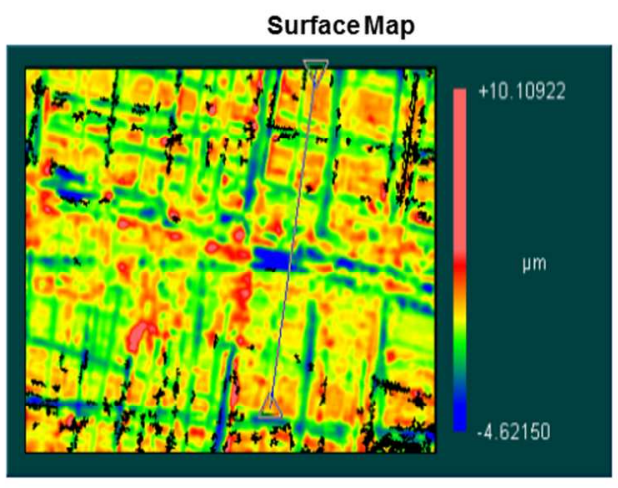

Surface Profile

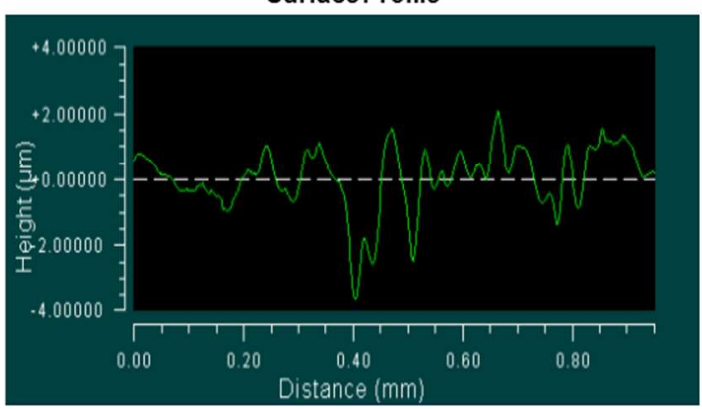

3D Model

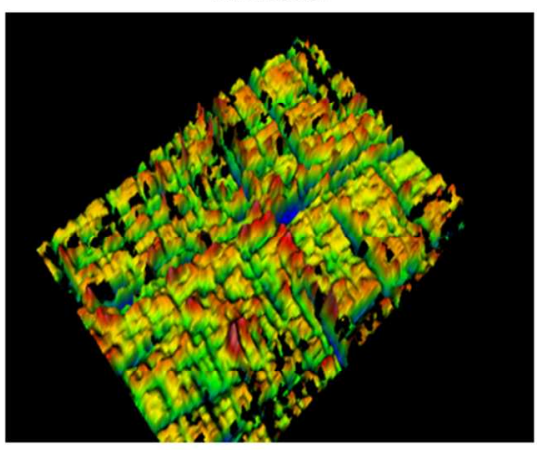

Intensity Map

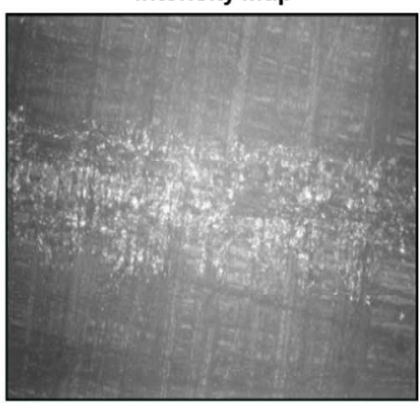

Figure S8. Interferometer data for Wire 1 before shrinking.

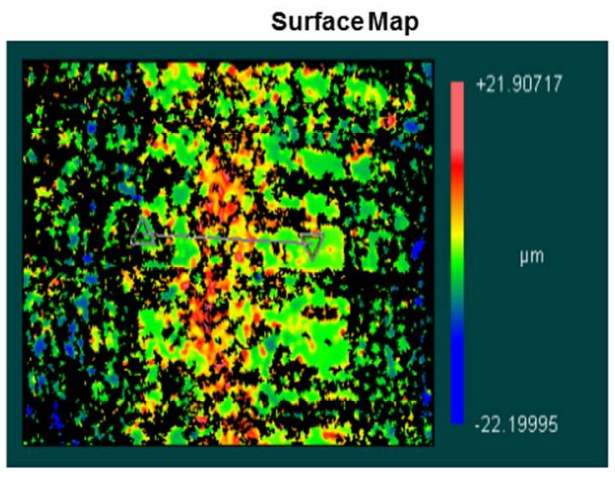

Surface Profile

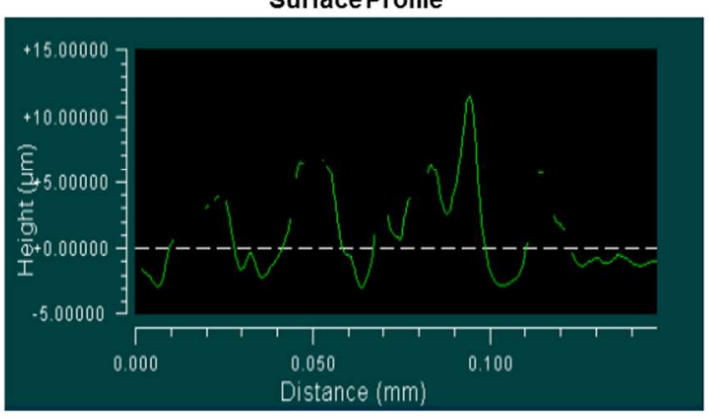

3D Model

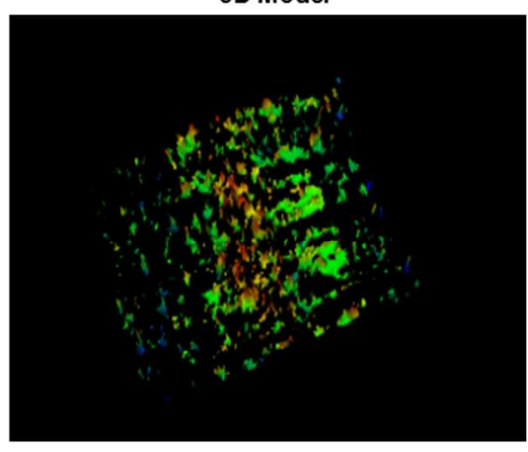

Intensity Map

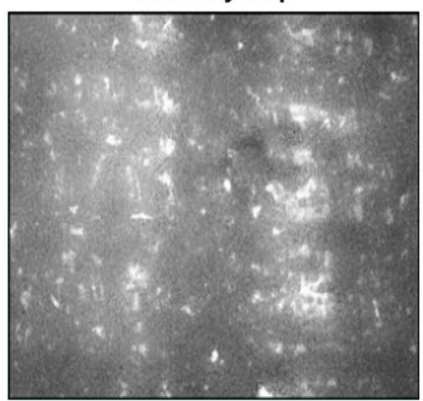

Figure S9. Interferometer data for Wire 1 after shrinking. 
Surface Map

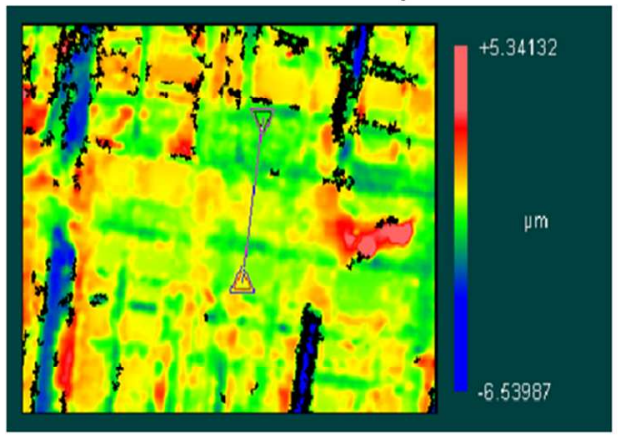

Surface Profile

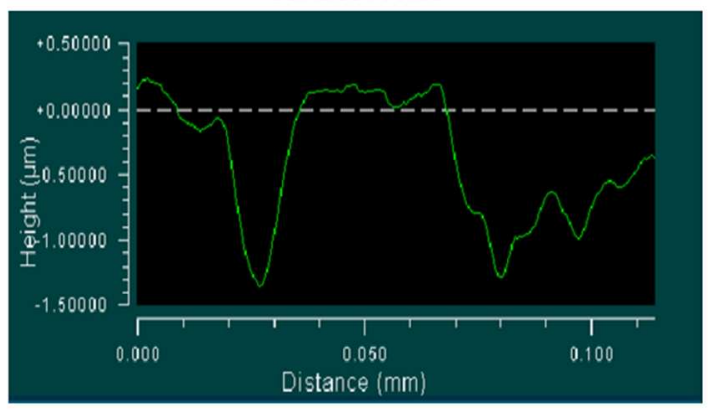

3D Model

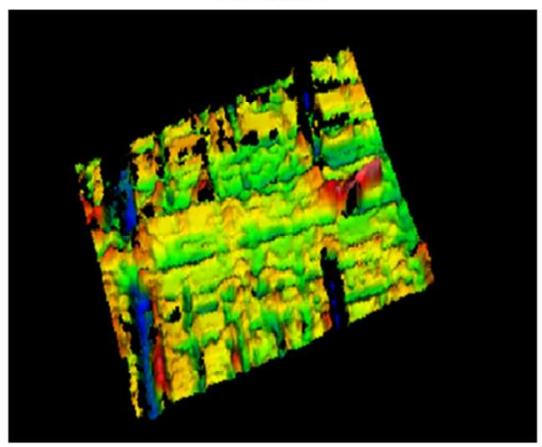

Intensity Map

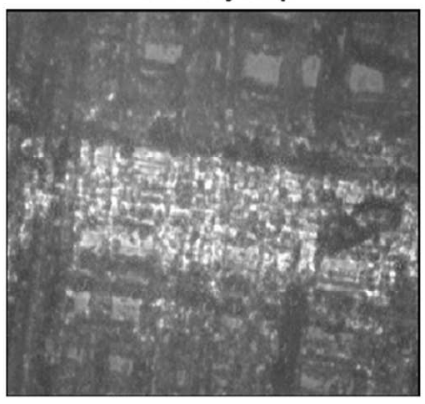

Figure S10. Interferometer data for Wire 2 before shrinking.

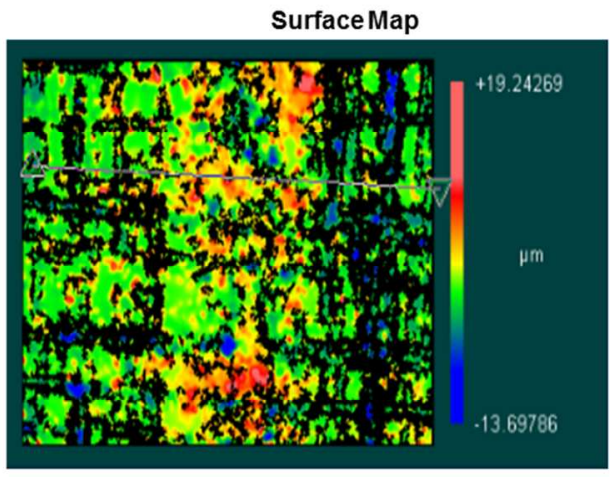

Surface Profile

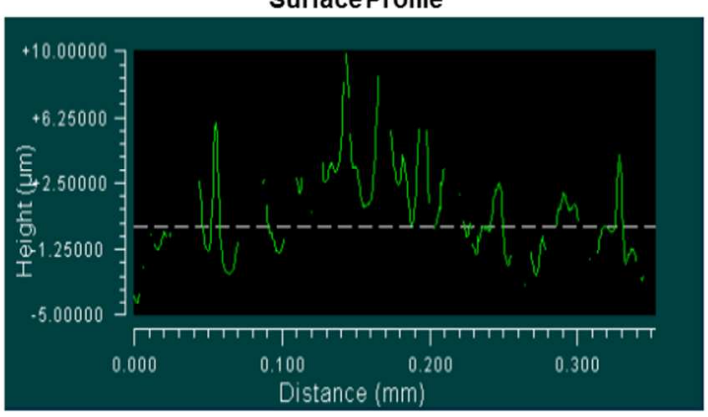

3D Model

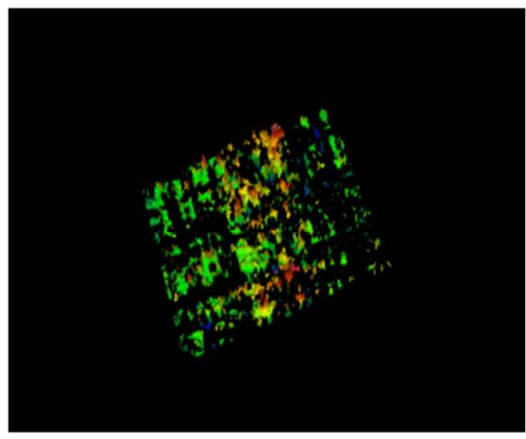

Intensity Map

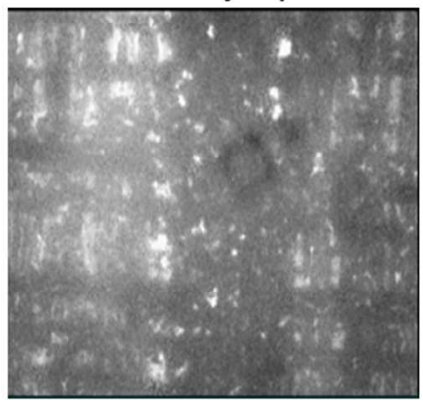

Figure S11. Interferometer data for Wire 2 after shrinking. 


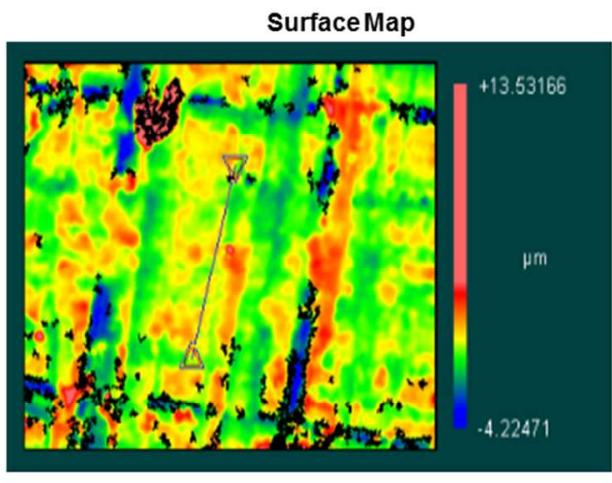

Surface Profile

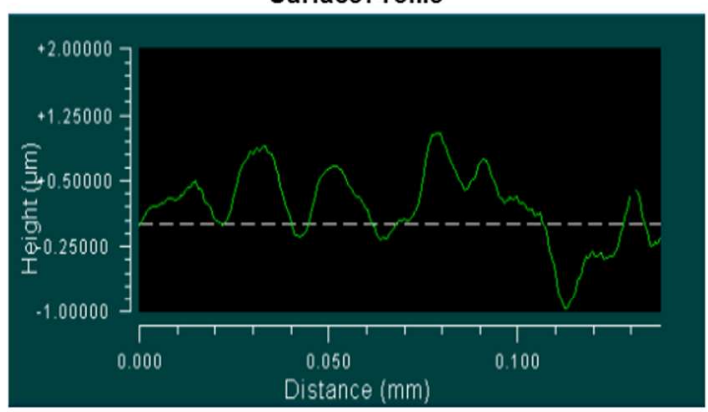

3D Model

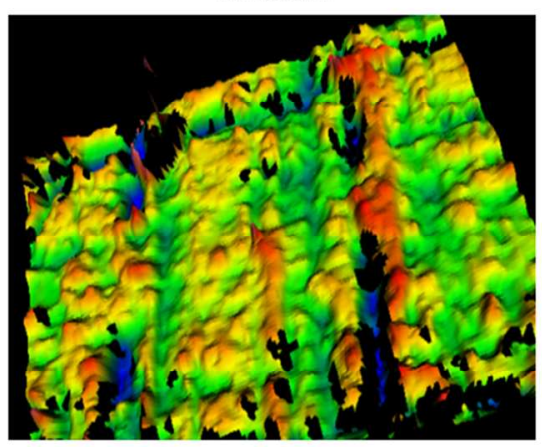

Intensity Map

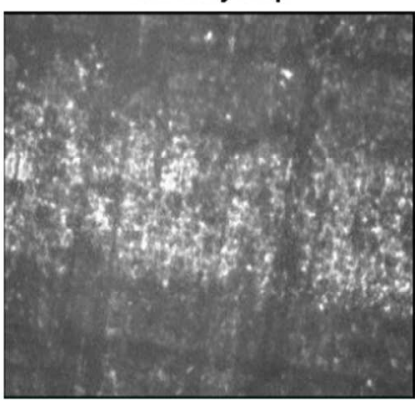

Figure S12. Interferometer data for Wire 3 before shrinking.

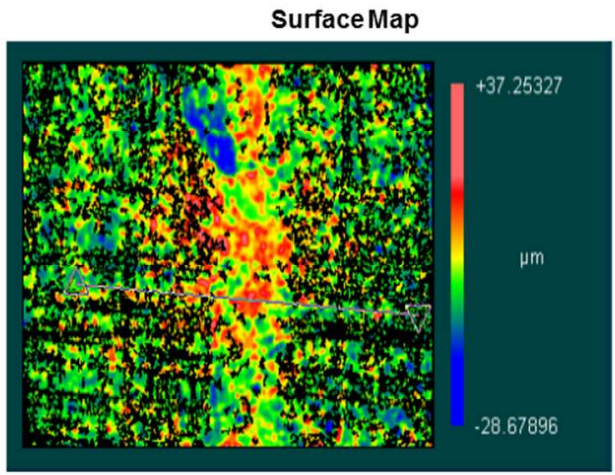

Surface Profile

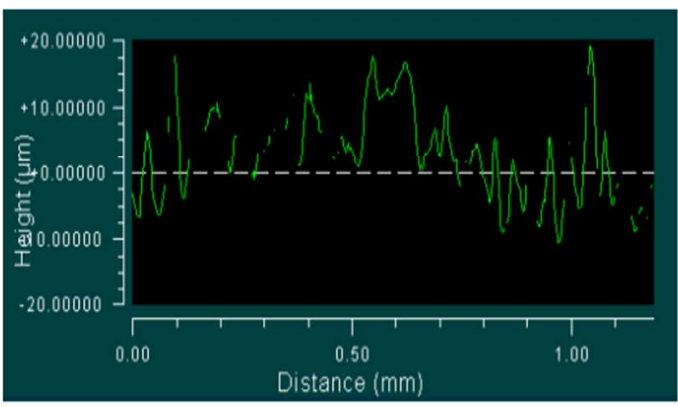

3D Model

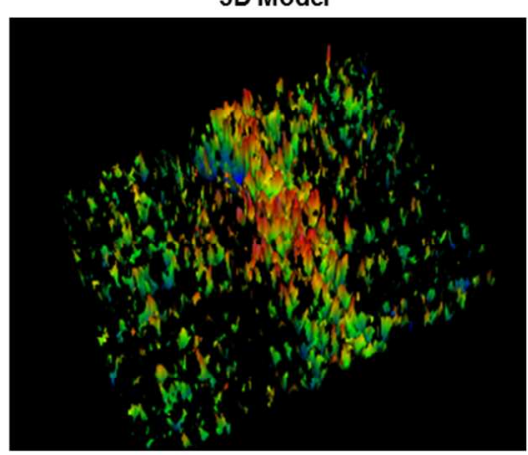

Intensity Map

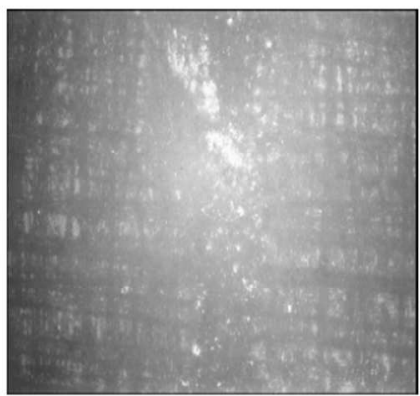

Figure S13. Interferometer data for Wire 3 after shrinking. 
Surface Map

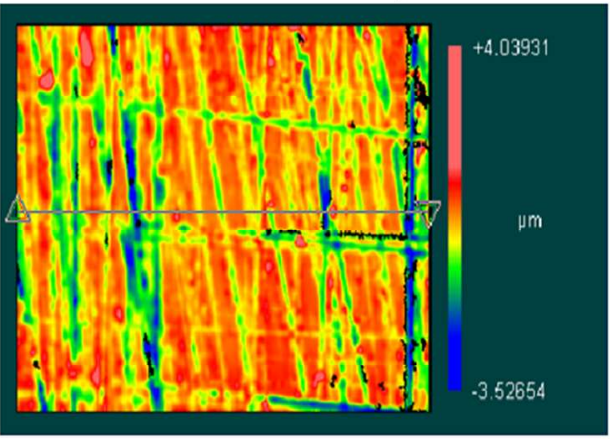

Surface Profile

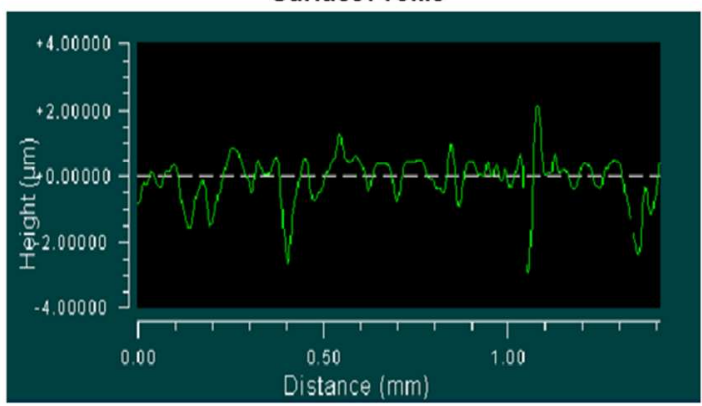

3D Mode

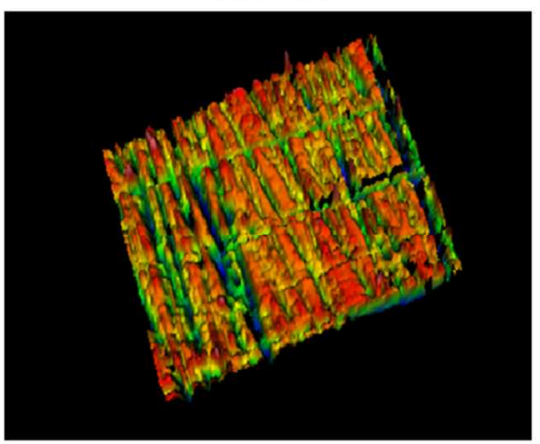

Intensity Map

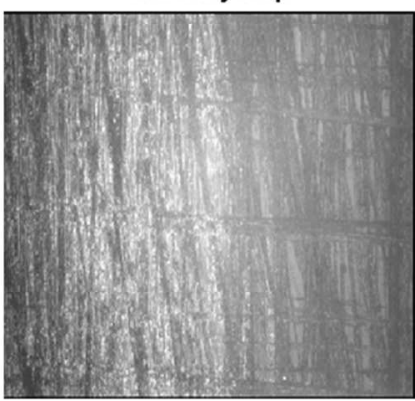

Figure S14. Interferometer data for electrode wire before shrinking.

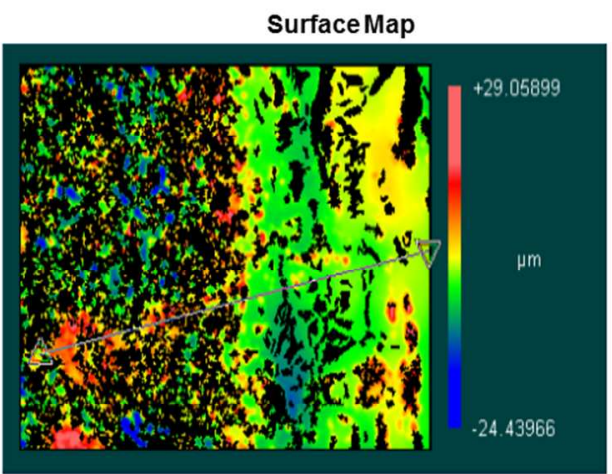

Surface Profile

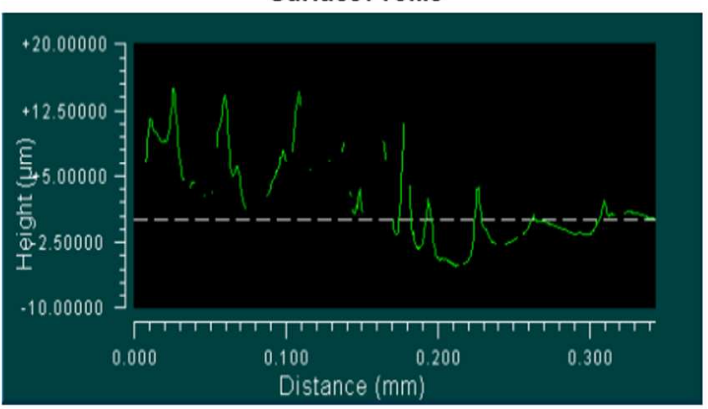

3D Model

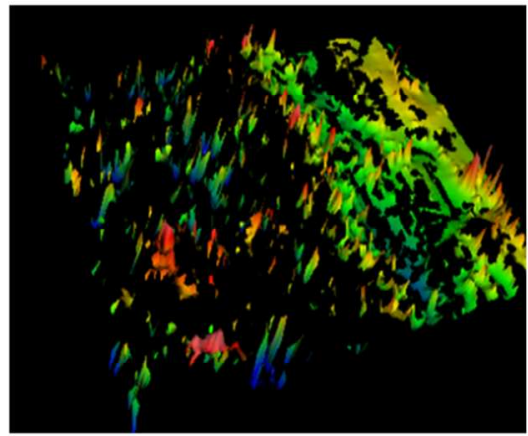

Intensity Map

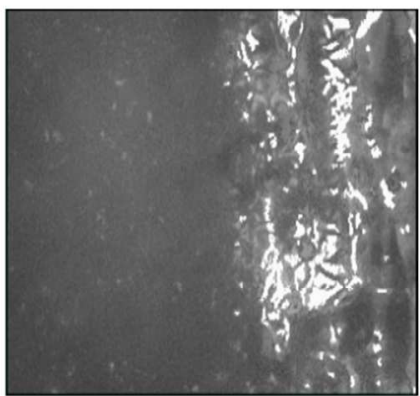

Figure S15. Interferometer data for electrode after shrinking. 


\section{4-Point Linear Probe Measurement of Resistivity}

A Singatone tungsten carbide four-point linear probe was employed to collect bulk conductance measurements of MOFs with a space between tips of $1.27 \mathrm{~mm}$. We calculated bulk conductance measurements $\sigma(\mathrm{S} / \mathrm{cm})$ of samples using Equation S1. Herein, I (A) is the current, $\mathrm{V}(\mathrm{V})$ represents voltage, $\mathrm{w}(\mathrm{cm})$ corresponds to the thickness of a circular sample pellet, $\mathrm{C}$ is a geometry correction factor (unitless) accounting for the diameter of a pellet sample, and $F$ (unitless) is the thickness correction factor that accounts for the thickness of a pellet sample.

$$
\sigma=\frac{I}{V \times w \times C \times F} \quad \text { Equation S1 }
$$

Using this method, we calculated bulk conductance for $\mathrm{Cu}_{3} \mathrm{HHTP}_{2} \mathrm{MOF}$ as $\left(7.6 \times 10^{-3}\right) \pm(0.3 \mathrm{x}$ $\left.10^{-3}\right) \mathrm{S} / \mathrm{cm}$, and for $\mathrm{Ni}_{3} \mathrm{HHTP}_{2}$ MOF as $\left(1.0 \times 10^{-2}\right) \pm\left(0.3 \times 10^{-2}\right) \mathrm{S} / \mathrm{cm}$. 


\section{Current/Voltage Plots}

Data for current/voltage plots collected using portable EmSTAT potentiostats.

A.
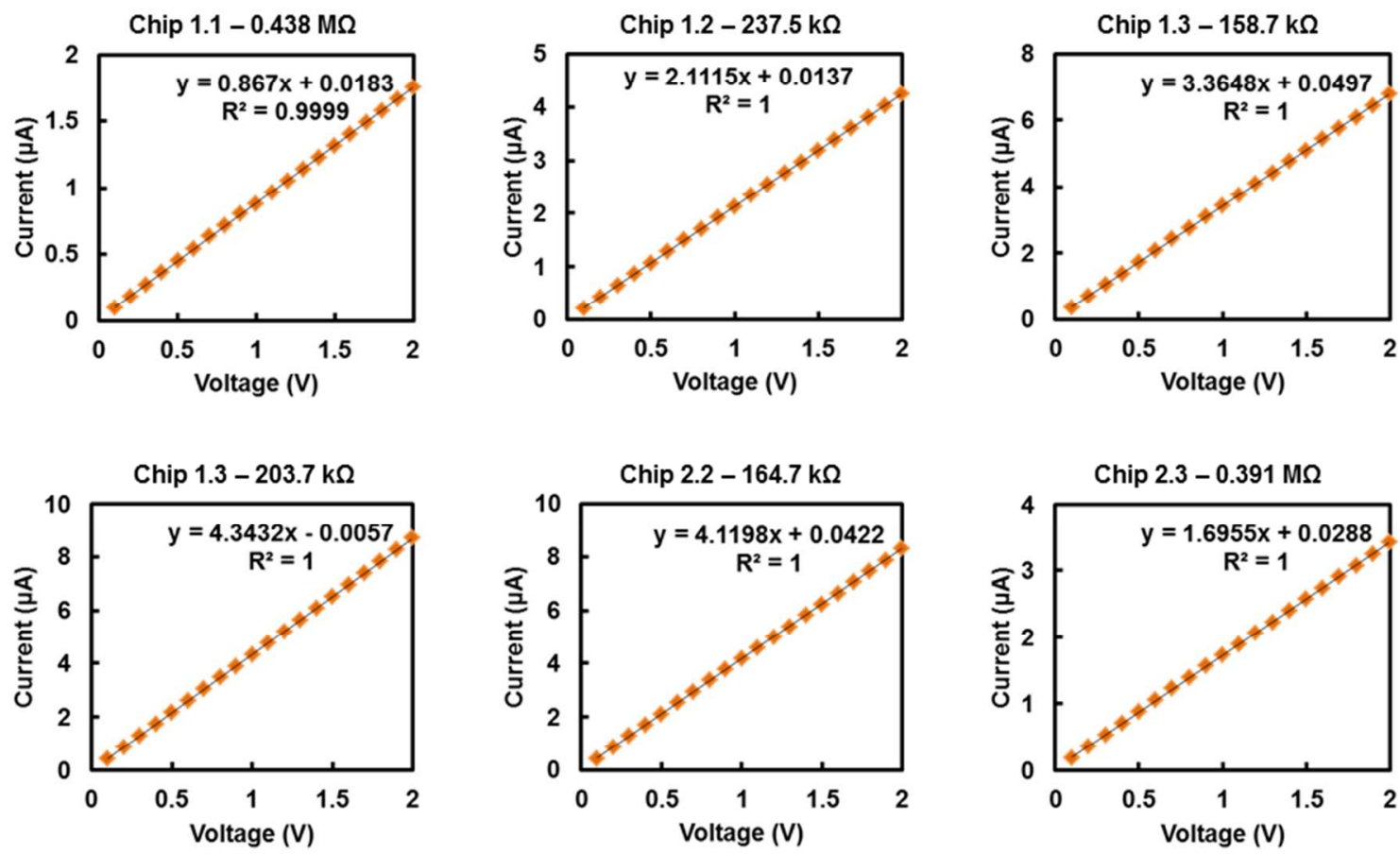

B.
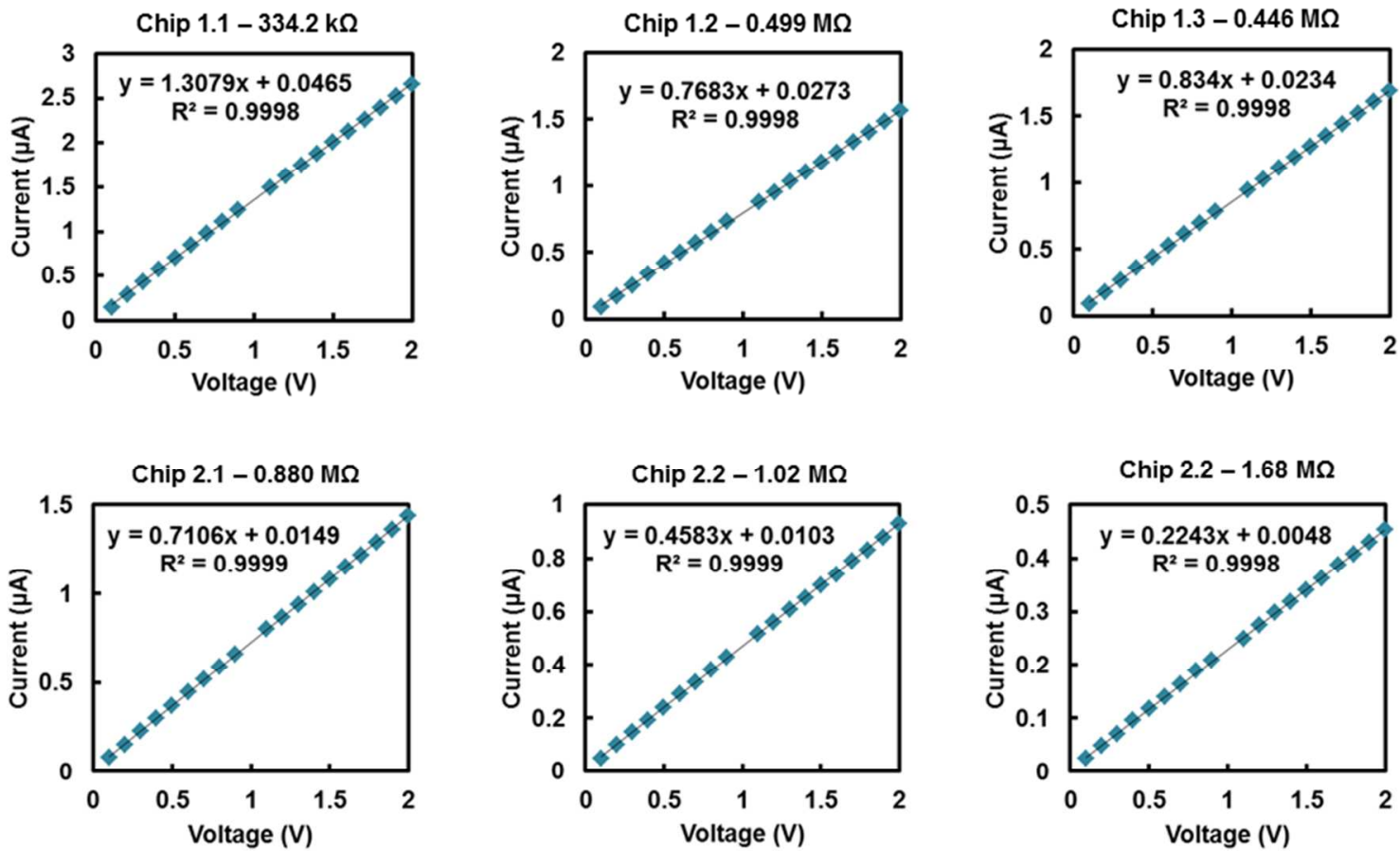

Figure S16. A) IV plots for six devices on two chips demonstrating Ohmic nature of contacts for $\mathrm{Cu}_{3} \mathrm{HHTP} \mathrm{POF}_{2}$, and B) IV plots for six devices on two chips demonstrating Ohmic nature of contacts for $\mathrm{Ni}_{3} \mathrm{HHTP}_{2} \mathrm{MOF}$. 


\section{Scanning Electron Microscopy of Devices}

Scanning electron microscopy of MOF-templated devices was obtained using a using a Hitachi TM3000 SEM with a $17.0 \mathrm{kV}$ beam with gaseous secondary electron (GSE) and backscattered secondary electron (BSE) detectors.
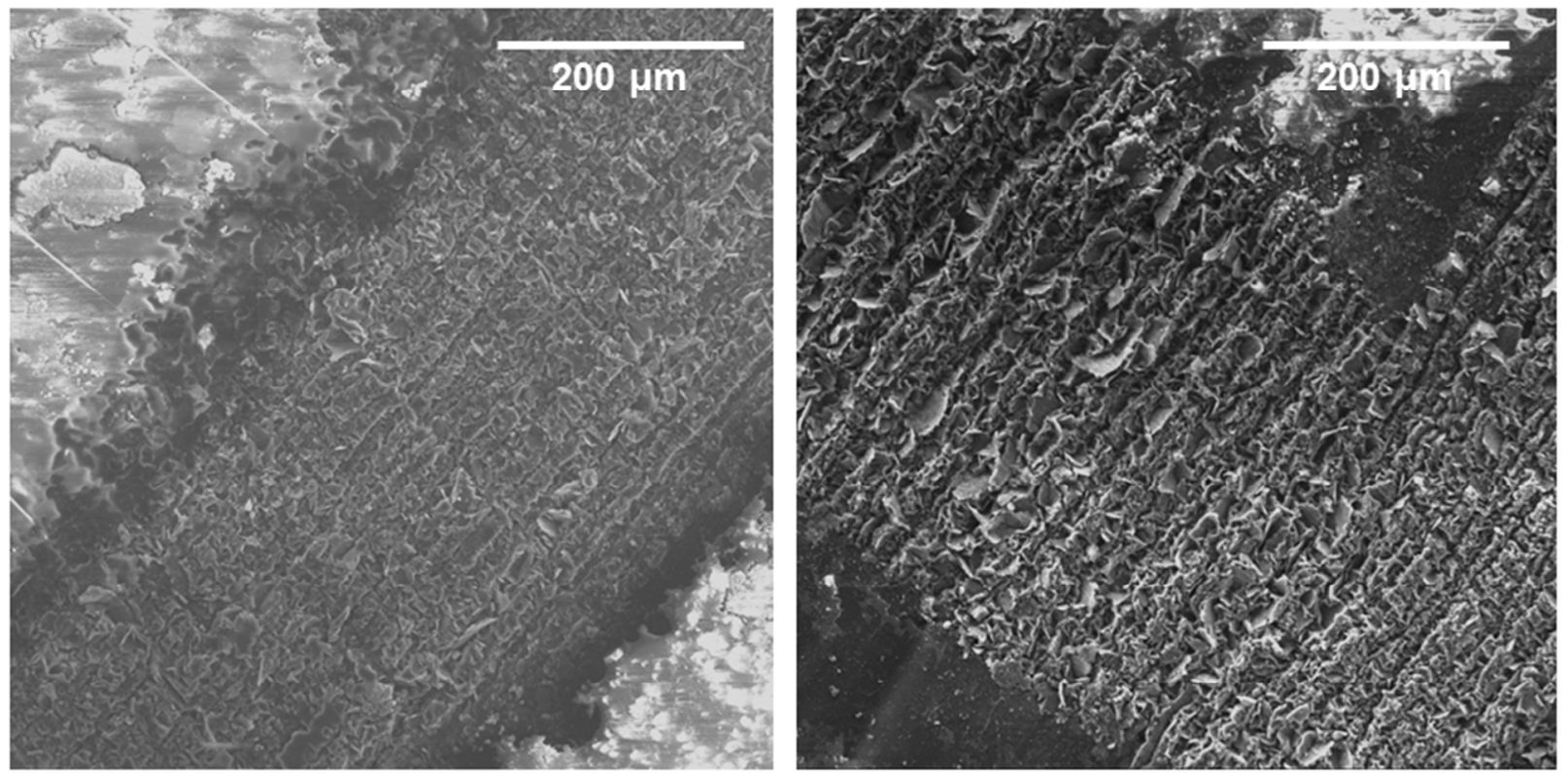

Figure S17. Scanning electron micrographs of a section of a graphite wire (left) and interdigitated electrode (right) drawn on a shrinkable polymer film substrate. These micrographs correspond to the fully drawn wires after substrate has been thermally miniaturized.
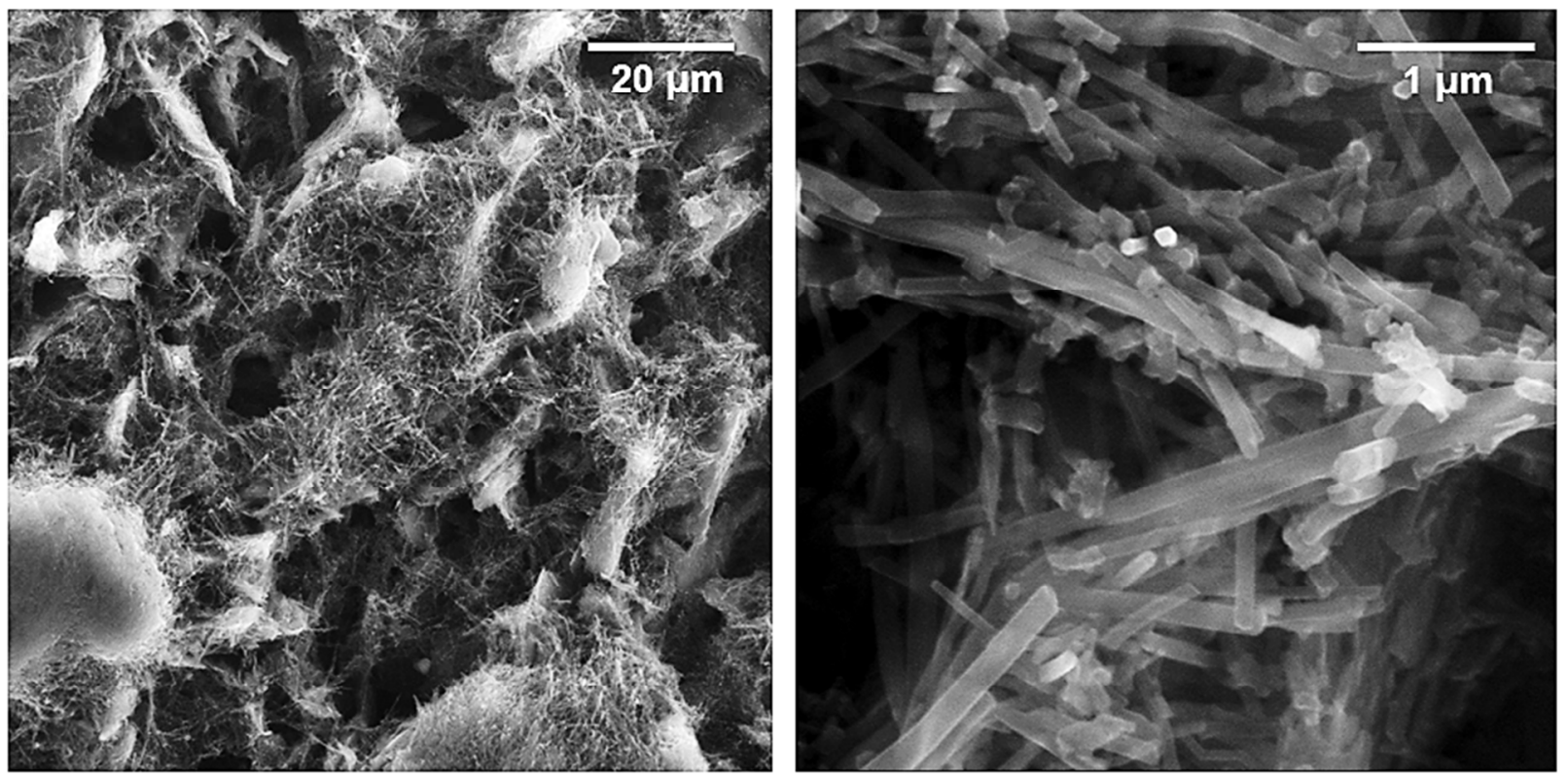

Figure S18. Scanning electron micrographs of $\mathrm{Cu}_{3} \mathrm{HHTP}_{2} \mathrm{MOF}$ templated on graphite wire at low (left) and high (right) magnification. 

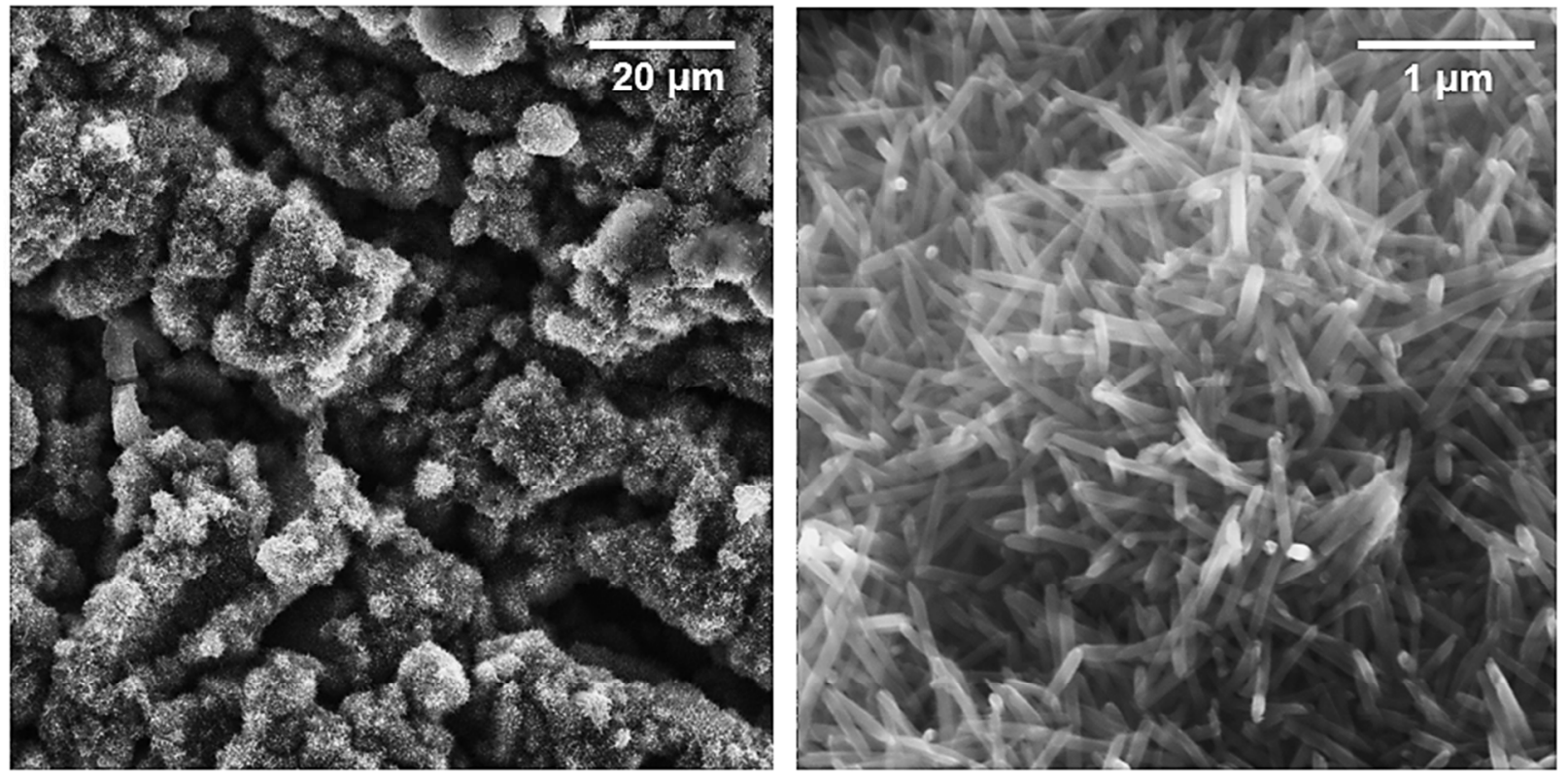

Figure S19. Scanning electron micrographs of $\mathrm{Ni}_{3} \mathrm{HHTP}_{2}$ MOF templated on graphite wire at low (left) and high (right) magnification.

\section{Energy Dispersive X-Ray Spectroscopy of Devices}

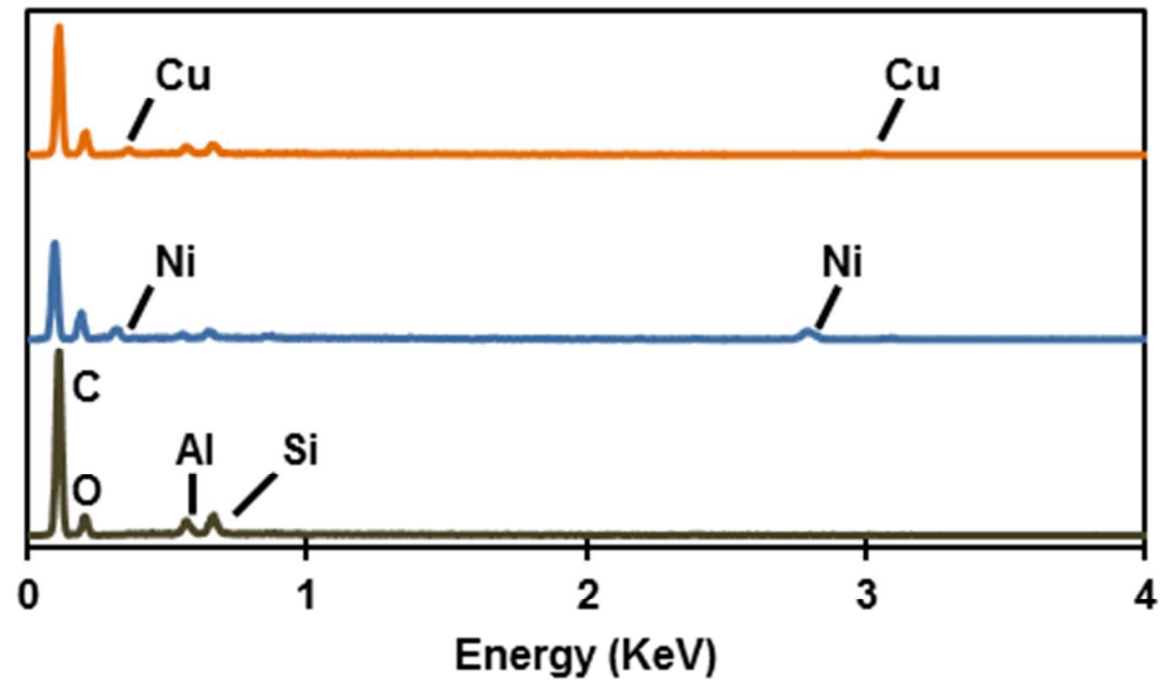

Figure S20. Energy dispersive spectroscopy of templated devices; graphite wire (gray), $\mathrm{Cu}_{3} \mathrm{HHTP}_{2} \mathrm{MOF}$ (orange) and $\mathrm{Ni}_{3} \mathrm{HHTP}_{2} \mathrm{MOF}$ (blue), from bottom to top. 


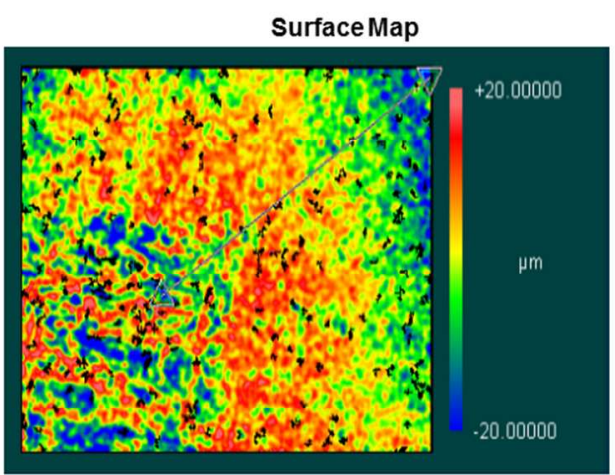

Surface Profile

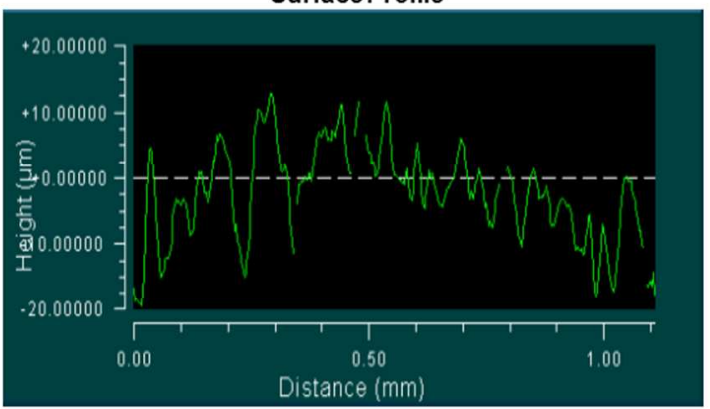

3D Model

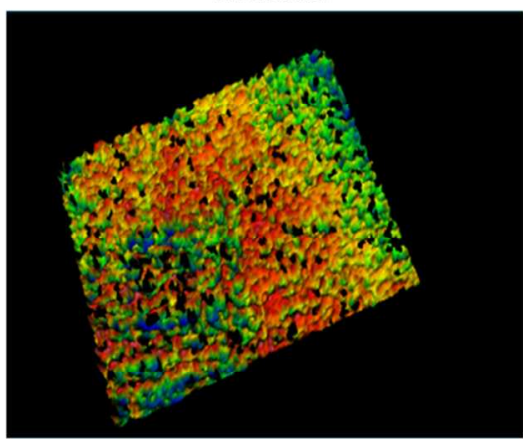

Intensity Map

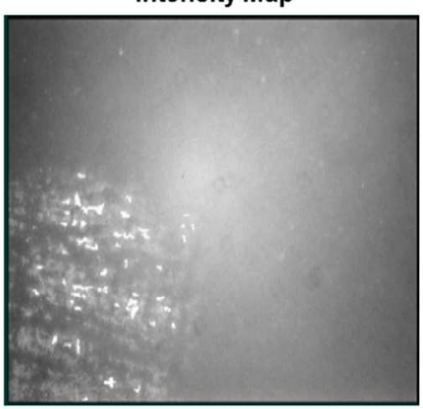

Figure S21. Representative interferometry data for $\mathrm{Cu}_{3} \mathrm{HHTP}_{2} \mathrm{MOF}$ film on electrode and polymer substrate.

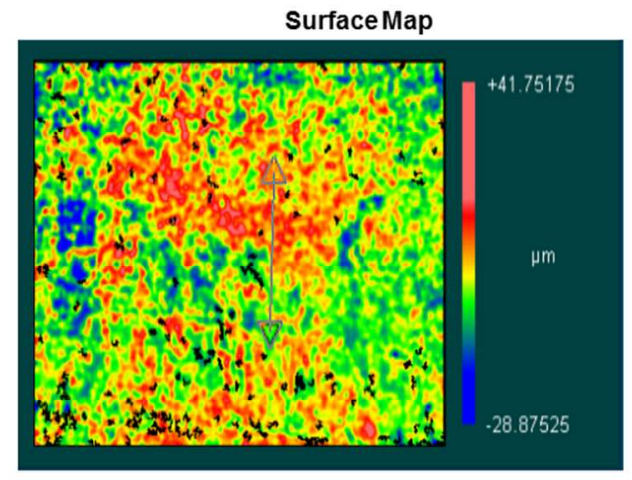

Surface Profile

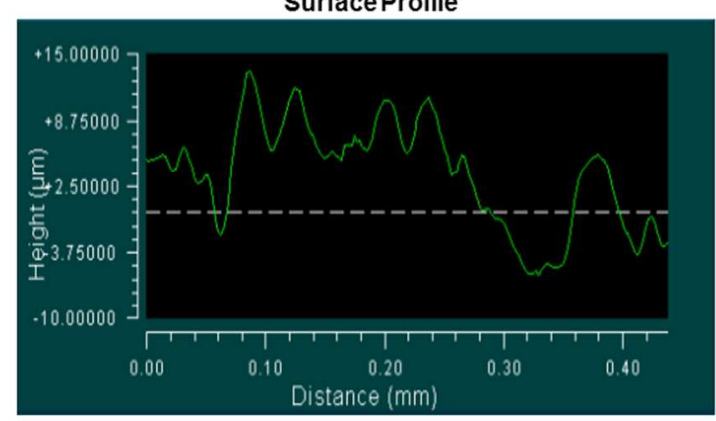

3D Model

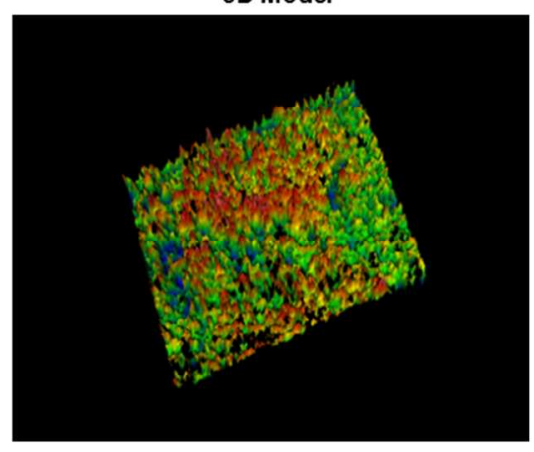

Intensity Map

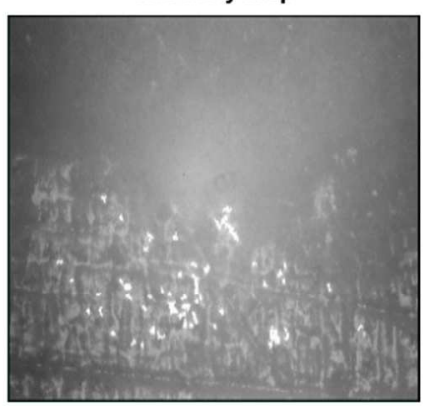

Figure S22. Representative interferometer data for $\mathrm{Ni}_{3} \mathrm{HHTP}_{2} \mathrm{MOF}$ film on electrode and polymer substrate. 


\section{Variance Device:Device and Batch:Batch}

Table S2. Representative normalized data from twelve devices (representing three total batches) to demonstrate the analyte-dependent average coefficients of variance between devices for $\mathrm{Cu}_{3} \mathrm{HHTP}_{2} \mathrm{MOF}$. Changes in conductance with respect to time are reported for the second, third, and fourth consecutive exposures (the first being highly variable) for each analyte at $80 \mathrm{ppm}$.

\begin{tabular}{|c|c|c|c|}
\hline \multirow{4}{*}{ Device } & \multicolumn{4}{|c|}{$\mathrm{Cu}_{3} \mathrm{HHTP}_{2}$} \\
\hline & $\mathbf{N H}_{3}$ & $\mathbf{N O}$ & $\mathbf{H}_{\mathbf{2}} \mathbf{S}$ \\
\hline $\mathbf{1}$ & 0.736779 & -0.92732 & 0.623391 \\
\hline $\mathbf{2}$ & 0.794393 & -1.2586 & 0.515639 \\
\hline $\mathbf{3}$ & 0.603428 & -1.8675 & 0.243045 \\
\hline $\mathbf{4}$ & 0.582497 & -1.9503 & 0.434844 \\
\hline $\mathbf{5}$ & 0.214913 & -1.6728 & 0.320706 \\
\hline $\mathbf{6}$ & 0.457704 & -1.6761 & 0.181745 \\
\hline $\mathbf{7}$ & 1.33484 & -2.07647 & 1.03665 \\
\hline $\mathbf{8}$ & 1.12045 & -2.01354 & 0.796764 \\
\hline $\mathbf{9}$ & 0.381826 & -1.88123 & 0.247556 \\
\hline $\mathbf{1 0}$ & 0.510533 & -2.38240 & 0.444015 \\
\hline $\mathbf{1 1}$ & 0.536831 & -2.34616 & 0.175636 \\
\hline $\mathbf{1 2}$ & 0.937467 & -2.04041 & 0.423231 \\
\hline $\begin{array}{c}\text { Coefficient of } \\
\text { variance for all } \\
\text { devices }\end{array}$ & $\mathbf{N H}_{\mathbf{3}}=\mathbf{4 7} \%$ & $\mathbf{N O}=\mathbf{2 3} \%$ & \multirow{2}{*}{$\mathbf{H}_{\mathbf{2}} \mathbf{S}=\mathbf{5 6} \%$} \\
\hline & & \multicolumn{2}{c}{} \\
\hline
\end{tabular}

Table S3. Representative normalized data from twelve devices (representing three total batches) to demonstrate the analyte-dependent average coefficients of variance between devices for $\mathrm{Ni}_{3} \mathrm{HHTP}_{2} \mathrm{MOF}$. Changes in conductance with respect to time are reported for the second, third, and fourth consecutive exposures (the first being highly variable) for each analyte at $80 \mathrm{ppm}$. Note that ammonia is not included for this MOF, as no statistically significant chemiresistive change in conductance was observed.

\begin{tabular}{|c|c|c|c|}
\hline \multicolumn{4}{|c|}{$\mathrm{Ni}_{3} \mathrm{HHTP}_{2}$} \\
\hline \multirow{2}{*}{ Device } & \multicolumn{3}{|c|}{ Average Exposure 3 x 80 ppm $\left(\Delta \mathrm{l} / \mathrm{l}_{\mathrm{o}}(\%)\right)$} \\
\hline & $\mathrm{NH}_{3}$ & NO & $\mathrm{H}_{2} \mathrm{~S}$ \\
\hline 1 & \multirow{12}{*}{$\begin{array}{c}\text { not applicable, } \\
\text { no response }\end{array}$} & -1.47646 & 4.11926 \\
\hline 2 & & -2.09025 & 3.33022 \\
\hline 3 & & -2.26250 & 6.24912 \\
\hline 4 & & -2.75507 & 2.70219 \\
\hline 5 & & -4.17193 & 4.38674 \\
\hline 6 & & -2.53260 & 3.69082 \\
\hline 7 & & -1.44776 & 3.35039 \\
\hline 8 & & -1.20580 & 4.77987 \\
\hline 9 & & -1.58202 & 4.30139 \\
\hline 10 & & -1.04096 & 3.78305 \\
\hline 11 & & -0.091150 & 4.39629 \\
\hline 12 & & 0.078632 & 5.354324 \\
\hline $\begin{array}{l}\text { Coefficient of } \\
\text { variance for all } \\
\text { devices }\end{array}$ & $\mathrm{NH}_{3}=\mathbf{N A}$ & NO $=69 \%$ & $\mathrm{H}_{2} \mathrm{~S}=23 \%$ \\
\hline
\end{tabular}




\section{Chemiresistive Sensing Results}

A.

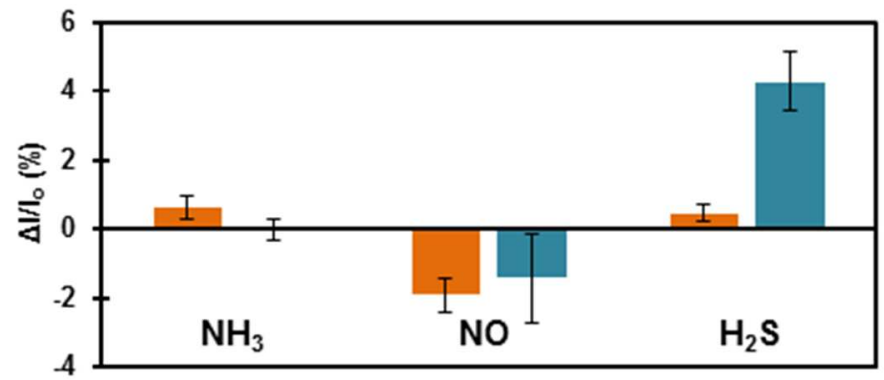

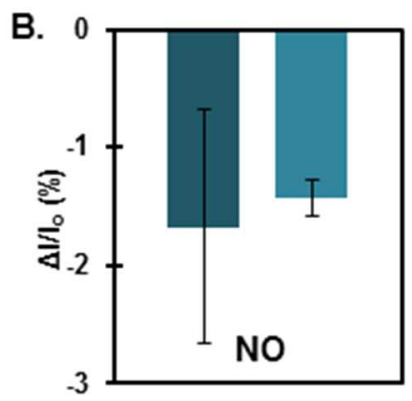

Figure S23. A) A plot summarizing average sensing responses of $\mathrm{Cu}_{3} \mathrm{HHTP}_{2}$ (orange) and $\mathrm{Ni}_{3} \mathrm{HTTP}_{2}$ (blue) MOFs exposed to gaseous analytes. Each device was exposed to $3 \times 80 \mathrm{ppm}$ analyte, and results are based on 12 representative devices for each MOF. B) A plot of average representative responses (3 devices) for three exposures of $\mathrm{Ni}_{3} \mathrm{HTTP}_{2}$ to $\mathrm{NO}$ before (left - dark blue) and after (right - blue) exposure to $\mathrm{H}_{2} \mathrm{~S}(4 \times 80 \mathrm{ppm}$ ), illustrating that the dosimetric analyte response to $\mathrm{H}_{2} \mathrm{~S}$ does not poison the subsequent response to NO.
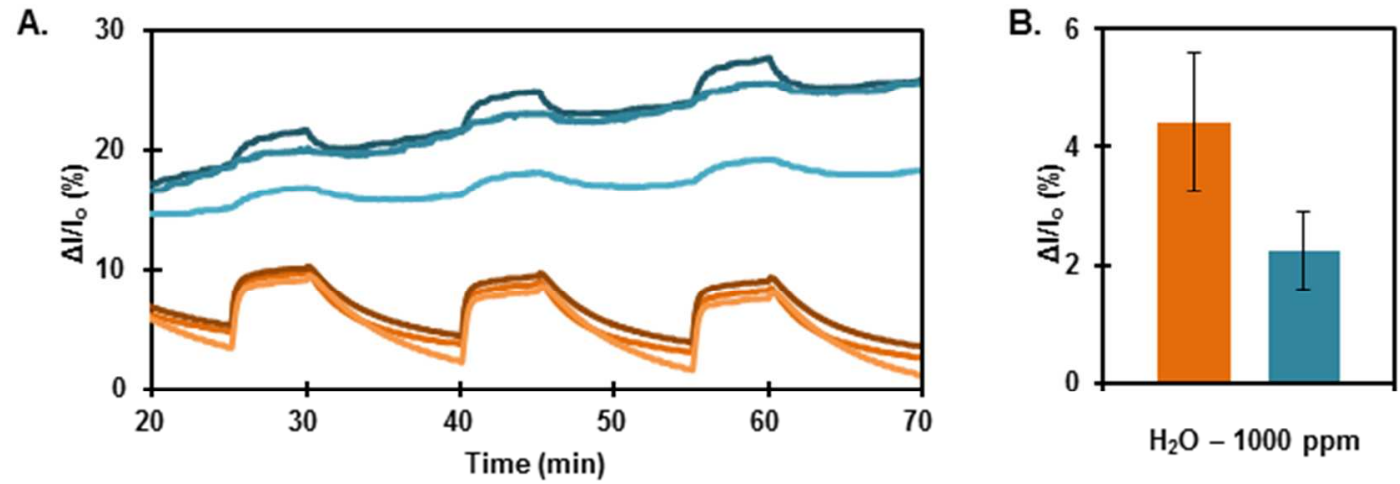

Figure S24. A) Representative sensing traces for $\mathrm{Cu}_{3} \mathrm{HHTP}_{2}$ (orange) and $\mathrm{Ni}_{3} \mathrm{HTTP}_{2}$ (blue) MOFs when exposed to $1000 \mathrm{ppm}$ water $(4 \times 1000 \mathrm{ppm})$, excluding the first exposure. B) Overall average sensing responses for water. $\mathrm{Cu}_{3} \mathrm{HHTP}_{2}$ (orange) and $\mathrm{Ni}_{3} \mathrm{HTTP}_{2}$ (blue) MOFs were exposed to $3 \times 1000 \mathrm{ppm}$ water, and results are based on 6 representative devices for each MOF.
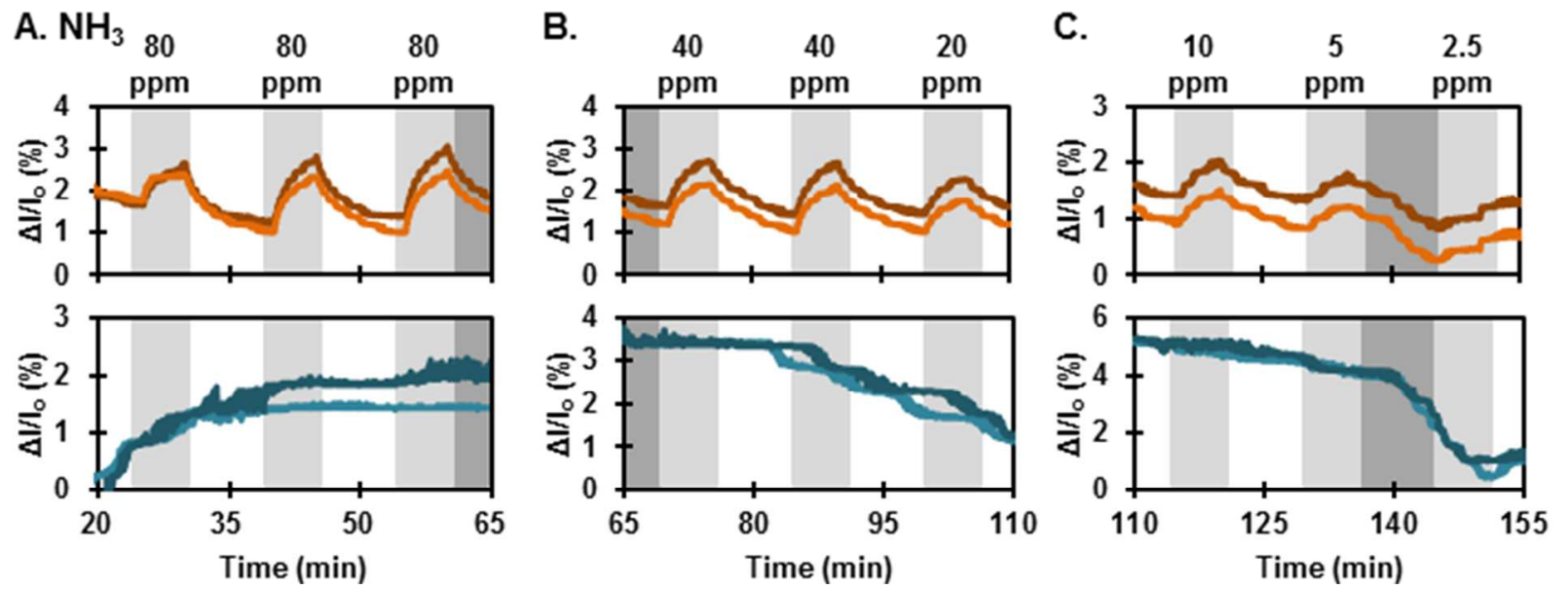

Figure S25. Representative sensing traces for a concentration dependence study with $\mathrm{NH}_{3}$, for $\mathrm{Cu}_{3} \mathrm{HHTP}_{2} \mathrm{MOF}$ (orange) and $\mathrm{Ni}_{3} \mathrm{HHTP}_{2} \mathrm{MOF}$ (blue), where dark gray bars correspond to a recovery phase change in dilution flow rate, and light gray bars correspond to a dose of $\mathrm{NH}_{3}$, for A) $3 \times 80$ ppm exposures, B) $2 \times 40$ and $1 \times 20$ ppm exposures, and C) $1 \times 10,1 \times 5$, and $1 \times 2.5$ ppm exposures. 

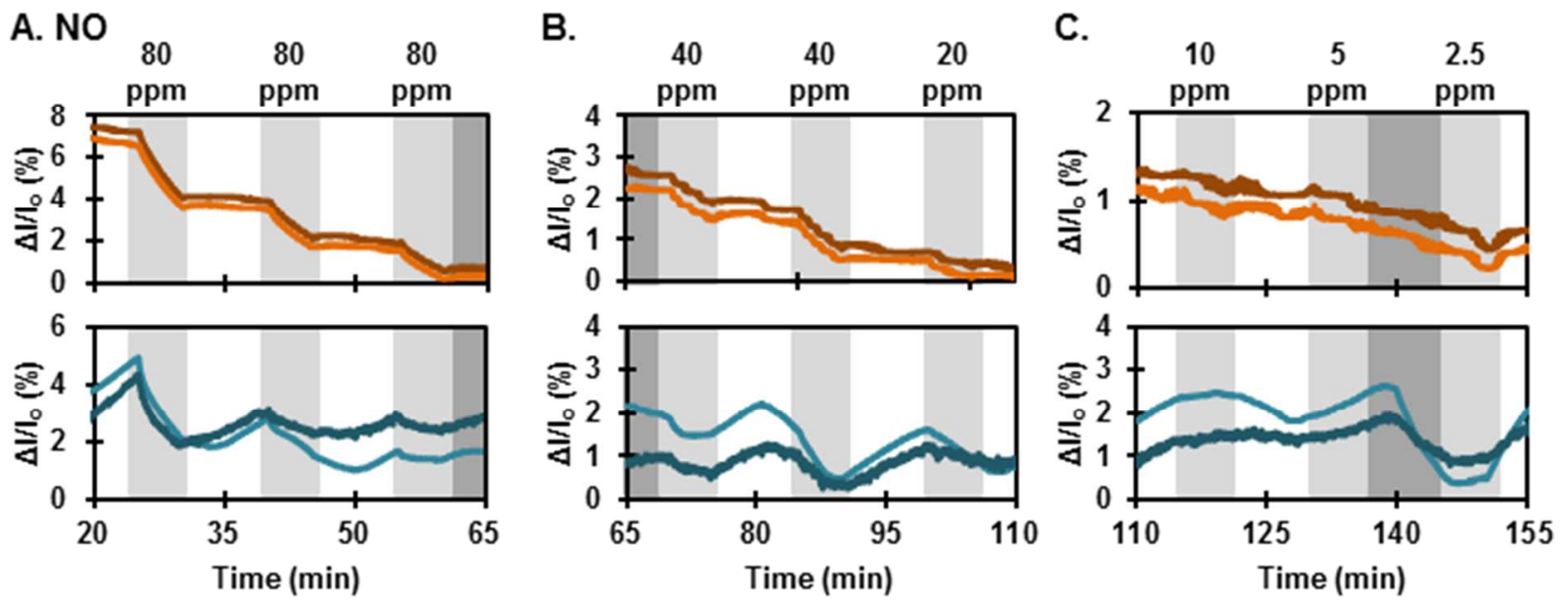

Figure S26. Representative sensing traces for a concentration dependence study with $\mathrm{NO}$, for $\mathrm{Cu}_{3} \mathrm{HHTP}_{2} \mathrm{MOF}$ (orange) and $\mathrm{Ni}_{3} \mathrm{HHTP}_{2} \mathrm{MOF}$ (blue), where dark gray bars correspond to a recovery phase change in dilution flow rate, and light gray bars correspond to a dose of NO, for A) $3 \times 80 \mathrm{ppm}$ exposures, B) $2 \times 40$ and $1 \times 20 \mathrm{ppm}$ exposures, and C) $1 \times 10,1 \times 5$, and $1 \times 2.5$ ppm exposures.
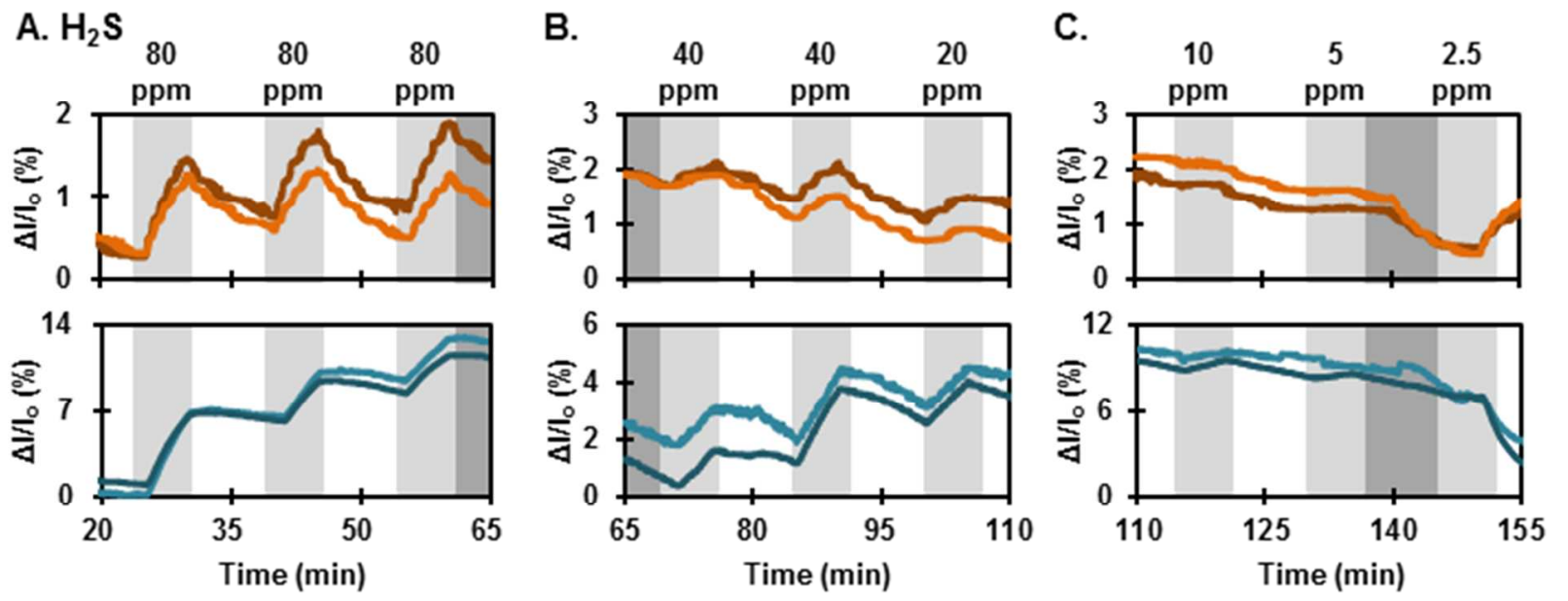

Figure S27. Representative sensing traces for a concentration dependence study with $\mathrm{H}_{2} \mathrm{~S}$, for $\mathrm{Cu}_{3} \mathrm{HHTP}_{2}$ MOF (orange) and $\mathrm{Ni}_{3} \mathrm{HHTP}_{2}$ MOF (blue), where dark gray bars correspond to a recovery phase change in dilution flow rate, and light gray bars correspond to a dose of $\mathrm{H}_{2} \mathrm{~S}$, for A) $3 \times 80$ ppm exposures, B) $2 \times 40$ and $1 \times 20 \mathrm{ppm}$ exposures, and C) $1 \times 10,1 \times 5$, and $1 \times 2.5$ ppm exposures. 


\section{Principle Component Analysis}

Table S4. Average sensory response for three arrays, excluding first exposures.

\begin{tabular}{|c|c|c|c|c|c|c|}
\hline \multirow{2}{*}{ Analyte } & \multicolumn{2}{|c|}{ Array \#1 $\left(\Delta \mathrm{l} / \mathrm{I}_{\mathrm{o}}\right)$} & \multicolumn{2}{|c|}{ Array \#2 $\left(\Delta \mathrm{I} / \mathrm{I}_{\circ}\right)$} & \multicolumn{2}{|c|}{ Array \#3 $\left(\Delta \mathrm{l} / \mathrm{I}_{\mathrm{o}}\right)$} \\
\hline & $\mathrm{Cu}_{3} \mathrm{HHTP}_{2}$ & $\mathrm{Ni}_{3} \mathrm{HHTP}_{2}$ & $\mathrm{Cu}_{3} \mathrm{HHTP}_{2}$ & $\mathrm{Ni}_{3} \mathrm{HHTP}_{2}$ & $\mathrm{Cu}_{3} \mathrm{HHTP}_{2}$ & $\mathrm{Ni}_{3} \mathrm{HHTP}_{2}$ \\
\hline $80 \mathrm{ppm} \mathrm{NH}$ & 1.33483 & -0.139110 & 1.12045 & 0.109591 & 0.381826 & 0.208558 \\
\hline 80 ppm NO & 1.06332 & 0.833328 & 0.963799 & -0.073126 & 0.548033 & -0.527189 \\
\hline $80 \mathrm{ppm} \mathrm{H} \mathrm{H}_{2} \mathrm{~S}$ & 0.615208 & 0.416664 & 0.481805 & -0.219378 & 0.265649 & 0.032653 \\
\hline $40 \mathrm{ppm} \mathrm{NH}_{3}$ & 0.327078 & 0.832661 & 0.387776 & -0.366215 & 0.232443 & -0.329164 \\
\hline 40 ppm NO & -2.07647 & -1.27783 & -2.01354 & -0.761624 & -1.88123 & -0.613629 \\
\hline $40 \mathrm{ppm} \mathrm{H} \mathrm{H}_{2} \mathrm{~S}$ & -0.886224 & -0.402250 & -0.921375 & -0.341389 & -0.827276 & -0.363059 \\
\hline $10 \mathrm{ppm} \mathrm{NH}$ & -0.208523 & 0.547423 & -0.233236 & 0.088912 & -0.264813 & 0.121020 \\
\hline 10 ppm NO & -0.182458 & 0.425690 & -0.175020 & 0.398426 & -0.248130 & 0.213720 \\
\hline $10 \mathrm{ppm} \mathrm{H} \mathrm{H}_{2} \mathrm{~S}$ & 1.03664 & 3.09562 & 0.796764 & 2.67804 & 0.247556 & 3.53936 \\
\hline 1000 ppm $\mathrm{H}_{2} \mathrm{O}$ & 0.662252 & 1.79075 & 0.392205 & 1.41651 & -0.103609 & 1.45083 \\
\hline
\end{tabular}

Table S5. Principle Component Scores for three arrays.

\begin{tabular}{|c|c|c|c|}
\hline \multicolumn{4}{|c|}{ Principle Component Scores } \\
\hline \multirow{11}{*}{ 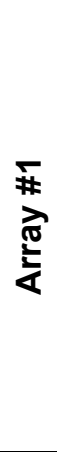 } & Analyte & PC1 (89\%) & PC2 (11\%) \\
\hline & $80 \mathrm{ppm} \mathrm{NH}{ }_{3}$ & -0.027954 & 1.17976 \\
\hline & 80 ppm NO & 0.467672 & 0.300154 \\
\hline & $80 \mathrm{ppm} \mathrm{H} \mathrm{H}_{2} \mathrm{~S}$ & -0.143816 & 0.277919 \\
\hline & $40 \mathrm{ppm} \mathrm{NH}$ & -0.053400 & -0.219974 \\
\hline & $40 \mathrm{ppm} \mathrm{NO}$ & -3.24531 & -0.427203 \\
\hline & $40 \mathrm{ppm} \mathrm{H} \mathrm{H}_{2} \mathrm{~S}$ & -1.78455 & -0.204694 \\
\hline & $10 \mathrm{ppm} \mathrm{\textrm {NH } _ { 3 }}$ & -0.633821 & -0.397008 \\
\hline & 10 ppm NO & -0.701468 & -0.292498 \\
\hline & $10 \mathrm{ppm} \mathrm{H} \mathrm{H}_{2} \mathrm{~S}$ & 2.04849 & -1.318399 \\
\hline & 1000 ppm $\mathrm{H}_{2} \mathrm{O}$ & 0.861074 & -0.660441 \\
\hline \multirow{11}{*}{$\begin{array}{l}\text { \# } \\
\text { 离 } \\
\frac{2}{4}\end{array}$} & Analyte & PC1 (76\%) & PC2 (24\%) \\
\hline & $80 \mathrm{ppm} \mathrm{NH}{ }_{3}$ & 0.321844 & 0.648779 \\
\hline & 80 ppm NO & 0.081873 & 0.667209 \\
\hline & $80 \mathrm{ppm} \mathrm{H} \mathrm{H}_{2} \mathrm{~S}$ & -0.362363 & 0.429804 \\
\hline & $40 \mathrm{ppm} \mathrm{NH}$ & -0.532681 & 0.467145 \\
\hline & 40 ppm NO & -2.51027 & -0.951247 \\
\hline & $40 \mathrm{ppm} \mathrm{H}_{2} \mathrm{~S}$ & -1.44084 & -0.476120 \\
\hline & $10 \mathrm{ppm} \mathrm{NH}_{3}$ & -0.649980 & -0.293800 \\
\hline & $10 \mathrm{ppm} \mathrm{NO}$ & -0.389956 & -0.471495 \\
\hline & $10 \mathrm{ppm} \mathrm{\textrm {H } _ { 2 } \mathrm { S }}$ & 1.90913 & -1.39627 \\
\hline & 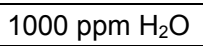 & 0.731029 & -0.790303 \\
\hline \multirow{11}{*}{ 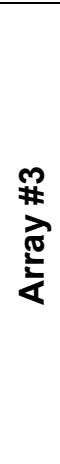 } & Analyte & PC1 (71\%) & PC2 (29\%) \\
\hline & $80 \mathrm{ppm} \mathrm{NH}$ & -0.127064 & 0.224035 \\
\hline & 80 ppm NO & -0.529789 & 0.861814 \\
\hline & $80 \mathrm{ppm} \mathrm{\textrm {H } _ { 2 } \mathrm { S }}$ & -0.333597 & 0.266270 \\
\hline & $40 \mathrm{ppm} \mathrm{NH}$ & -0.612921 & 0.498632 \\
\hline & 40 ppm NO & -2.30866 & -0.794813 \\
\hline & $40 \mathrm{ppm} \mathrm{H} \mathrm{H}_{2} \mathrm{~S}$ & -1.38622 & -0.226734 \\
\hline & $10 \mathrm{ppm} \mathrm{NH}$ & -0.646206 & -0.171308 \\
\hline & 10 ppm NO & -0.568859 & -0.225061 \\
\hline & $10 \mathrm{ppm} \mathrm{\textrm {H } _ { 2 } \mathrm { S }}$ & 2.13323 & -2.22614 \\
\hline & 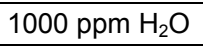 & 0.408098 & -0.997635 \\
\hline
\end{tabular}


XVIII. Infrared Analysis of Shrinkable Polymer Films

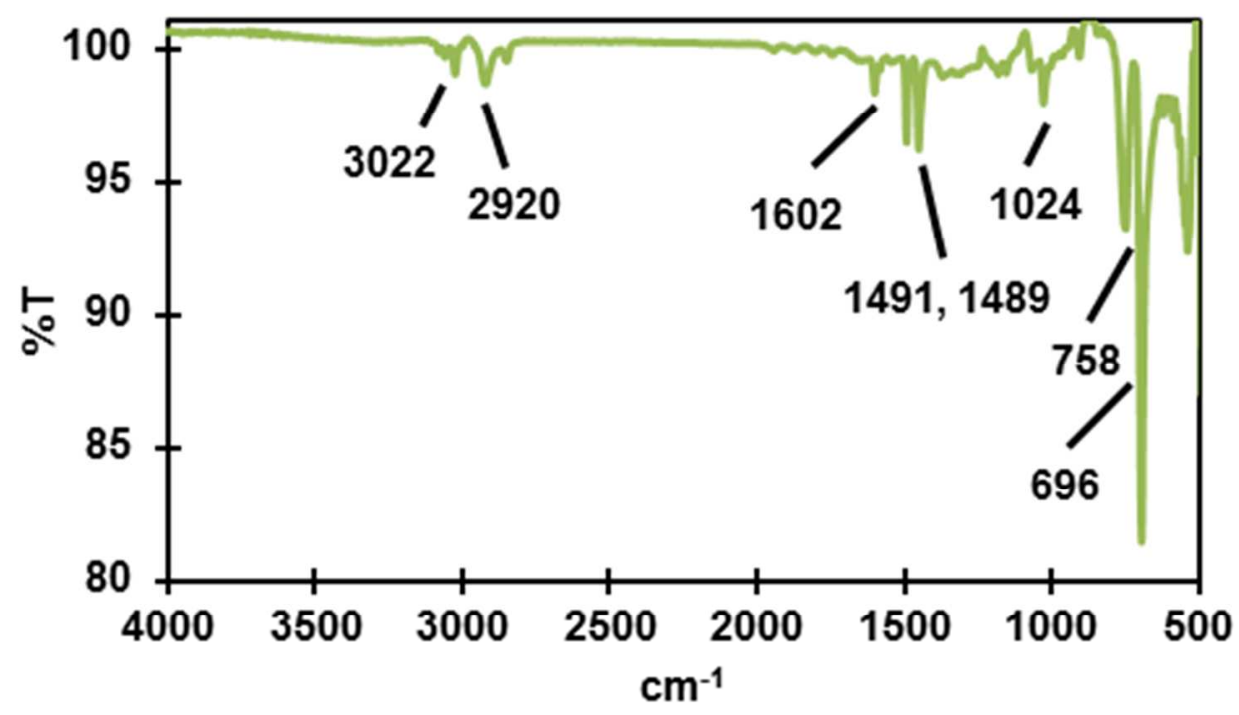

Figure S28. Infrared spectrum of polystyrene-based shrinkable polymer film with major stretches labeled. Fingerprint region is omitted.

Table S6. Summary of experimental high intensity infrared stretches for commercial shrinkable polymer film compared to reported strong vibrations for pure polystyrene.

\begin{tabular}{|c|c|c|}
\hline $\begin{array}{l}\text { Experimental } \\
\text { IR frequency } \\
\left(\mathrm{cm}^{-1}\right)\end{array}$ & $\begin{array}{c}\text { Reported } \\
\text { polystyrene } \\
\text { stretches }\left(\mathrm{cm}^{-1}\right)^{a, b}\end{array}$ & Assignment $^{b}$ \\
\hline 3022 & 3029 & aromatic \\
\hline 2920 & 2923 & aromatic/alkyl \\
\hline 1602 & 1602 & aromatic \\
\hline 1491,1489 & 1493 & alkyl \\
\hline 1024 & 1027 & $\begin{array}{l}\text { hydrogen- } \\
\text { bending }\end{array}$ \\
\hline 758 & 760 & aromatic \\
\hline 696 & 700 & aromatic \\
\hline
\end{tabular}

\section{Supporting References}

(S1) Composition of film is a proprietary trade secret. See Safety Data sheet GRX1275. http://www.grafixplastics.com/shrink.asp

(S2) Liang, C. Y.; Krimm, S. Infrared Spectra of High Polymers. VI. Polystyrene. J. Poly. Sci. 1958, 27, $241-254$. 